\title{
PALOMAR/LAS CAMPANAS IMAGING ATLAS OF BLUE COMPACT DWARF GALAXIES. II. SURFACE PHOTOMETRY AND THE PROPERTIES OF THE UNDERLYING STELLAR POPULATION
}

\author{
A. GiL de PAZ ${ }^{1}$ and B. F. MAdore ${ }^{1,2}$ \\ Received 2004 May 12; accepted 2004 October 21
}

\begin{abstract}
We present the results from an analysis of surface photometry of $B, R$, and $\mathrm{H} \alpha$ images of a total of 114 nearby galaxies $\left(v_{\text {helio }}<4000 \mathrm{~km} \mathrm{~s}^{-1}\right)$ drawn from the Palomar/Las Campanas Imaging Atlas of blue compact dwarf (BCD) galaxies. Surface brightness and color profiles for the complete sample have been obtained. We determine the exponential and Sérsic profiles that best fit the surface brightness distribution of the underlying stellar population detected in these galaxies. We also compute the $(B-R)$ color and total absolute magnitude of the underlying stellar population and compared them to the integrated properties of the galaxies in the sample. Our analysis shows that the $(B-R)$ color of the underlying population is systematically redder than the integrated color, except in those galaxies where the integrated colors are strongly contaminated by line and nebularcontinuum emission. We also find that galaxies with relatively red underlying stellar populations [typically $(B-R) \geq 1 \mathrm{mag}$ ] show structural properties compatible with those of dwarf elliptical galaxies (i.e., a smooth light distribution, fainter extrapolated central surface brightness, and larger scale lengths than BCD galaxies with blue underlying stellar populations). At least $\sim 15 \%$ of the galaxies in the sample are compatible with being dwarf elliptical (dE) galaxies experiencing a burst of star formation. For the remaining BCD galaxies in the sample we do not find any correlation between the recent star formation activity and their structural differences with respect to other types of dwarf galaxies.
\end{abstract}
Subject headings: atlases — galaxies: dwarf — galaxies: evolution — galaxies: fundamental parameters — galaxies: photometry - galaxies: starburst
Online material: color figures, extended figures, machine-readable tables

\section{INTRODUCTION}

The vast majority of the blue compact dwarf (BCD) galaxies are currently forming stars at a very high rate, as suggested by the large fraction of them ( $>93 \%$; Gil de Paz et al. 2003) showing significant $\mathrm{H} \alpha$ emission (EW > $20 \AA$ ). The star formation rate (SFR) derived for these galaxies ranges up $\sim 10 M_{\odot}$ $\mathrm{yr}^{-1}$ (Fanelli et al. 1988; Gil de Paz et al. 2003). This relatively high SFR, combined with a moderate gas content, implies gasconsumption timescales of $\sim 10^{9} \mathrm{yr}$, which are much shorter than the age of the universe.

This high SFR, combined with the low present-day metal abundances (see e.g., Hunter \& Hoffman 1999), led Searle et al. (1973) to suggest that either these objects are intrinsically young galaxies or that they have had an episodic star formation history involving very short bursts of star formation followed by long quiescent periods. It is now widely accepted that most of these objects possess a relatively evolved underlying stellar population (USP) associated with their low surface brightness (LSB) envelopes, i.e., they are not exclusively young galaxies (Schulte-Ladbeck et al. 1999, 2001; Drozdovsky et al. 2001; Crone et al. 2000, 2002; Gil de Paz et al. 2000a, 2000b; Papaderos et al. 1996a, 1996b). Despite considerable recent progress, the properties of this USP are still poorly known. Although the presence of an evolved population supports a relatively episodic star formation history, it has been recently shown that these episodes of star formation may, in fact, last as

\footnotetext{
${ }^{1}$ The Observatories, Carnegie Institution of Washington, 813 Santa Barbara Street, Pasadena, CA 91101; agpaz@ociw.edu.

2 NASA/IPAC Extragalactic Database, California Institute of Technology, MS 100-22, Pasadena, CA 91125; barry@ipac.caltech.edu.
}

long as $10^{8} \mathrm{yr}$ (Crone et al. 2002; Papaderos et al. 2002). However, it is still necessary that the SFR in BCDs had been much lower in the past (and for extended periods of time) as compared to today. It has been suggested that, even during the so-called quiescent phases, star formation could have taken place at a very low level, at a rather continuous rate (Legrand 2000).

The following questions naturally arise: What did BCD galaxies look like during these long periods of time of low (or null) star formation activity? Were they similar to local dwarf elliptical galaxies today? and, is there a single evolutionary model that can include all phases and types of dwarf galaxies?

Current knowledge bearing on these questions comes mainly from the analysis of the surface brightness profiles of the USP in BCDs and a comparison of the profiles of dwarf irregular and dwarf elliptical galaxies (Papaderos et al. 1996a; Noeske et al. 2000; Cairós et al. 2003). These results seem to suggest that the LSB envelopes associated with the USP of BCD galaxies are more compact and have a higher central surface brightness than those seen in $\mathrm{dI}$ and $\mathrm{dE}$ galaxies. Some additional clues have been recently provided by the study of the dynamics of BCD neutral hydrogen halos (van Zee et al. 2001; Tajiri \& Kamaya 2002). These authors have shown that the suggested evolution between $\mathrm{BCDs}$ and $\mathrm{dE}$ is difficult because of the relatively low efficiency of stellar feedback in potentially removing the $\mathrm{H} \mathrm{I}$ envelopes from these galaxies. It is worth emphasizing here that in all of these previous studies the number of objects under consideration was quite limited and no definitive conclusions could be drawn concerning existence or not of a unified evolutionary model for dwarf galaxies.

In Paper I (Gil de Paz et al. 2003) we described the integrated properties of a sample of 114 nearby galaxies in the Palomar/ 
Las Campanas Imaging Atlas of BCD galaxies. In this, the second paper of the series, we analyze the surface brightness profiles of the sample in $B$ and $R$ bands and in the light of $\mathrm{H} \alpha$. The morphological information derived, along with the properties of the USP detected in these galaxies, is now compared with the integrated properties measured in Paper I.

In $\S 2$ we briefly describe the sample and the observations carried out within the Atlas. The procedures used to derive the surface brightness and color profiles and the corresponding bestfitting exponential and Sérsic laws are described in $\S \S 3.1$ and 3.2 , respectively. The analysis of the surface brightness profiles is presented in $\S 4.1$. We describe the structural properties, color, and luminosity of the USP of these galaxies in $\S 4$.2. Finally, we discuss the implications of this study regarding the existence of possible evolutionary links between BCDs and dwarf elliptical galaxies $(\S 4.3)$, and the possible impact of the recent star formation on the structural properties of BCDs ( $\S 4.4)$. Our conclusions are summarized in $\oint 5$.

\section{SAMPLE AND OBSERVATIONS}

The original sample described in Paper I consisted of 114 galaxies. Of these, 105 galaxies were finally classified as BCD galaxies according to the set of quantitative criteria set out in that paper. The criteria include considerations about the galaxy's peak surface brightness, and the color at the position of this peak, along with an upper limit in the galaxy integrated absolute $K$-band luminosity (i.e., stellar mass). In this paper we have removed IC 10 from the sample of BCDs because of its very high Galactic extinction, which makes the intrinsic luminosity and colors of the USP highly uncertain. Although we computed the surface brightness profiles for all the 114 galaxies, our conclusions are based exclusively on the analysis of the properties of the final 104 BCD galaxies in the Atlas.

In Paper I we also presented an extensive description of the observations. Briefly, we observed 86 of the 114 Atlas galaxies at the Palomar 60 inch (1.524 m) telescope using a $2048 \times$ $2048 \mathrm{CCD}$ in $B, R$, and the appropriately redshifted $\mathrm{H} \alpha$ narrowband filter $(\Delta \lambda \sim 20 \AA$; see Paper I for a description of the filters used). Typical exposure times were $900 \mathrm{~s}$ in $B, 2700 \mathrm{~s}$ in $R$, and $5400 \mathrm{~s}$ in $\mathrm{H} \alpha$. The remaining 28 galaxies were observed at the Las Campanas Observatory 100 inch (2.54 m) (du Pont) telescope using a similar $2048 \times 2048 \mathrm{CCD}$. In this case the exposure times were $900 \mathrm{~s}$ in $B$ and $R$, and $1800 \mathrm{~s}$ in narrowband $\mathrm{H} \alpha(\Delta \lambda \sim 65 \AA)$.

\section{ANALYSIS}

\subsection{Surface Brightness and Color Profiles}

We used the flux-calibrated images of our Atlas to derive the surface brightness profiles of the sample. We first interactively eliminated foreground and background sources by interpolation using the same criteria as in Paper I (see also Gil de Paz et al. 2000b). Then, we fitted the galaxy isophotes using the iterative method described by Jedrzejewski (1987) within the IRAF task ELLIPSE. The output of this task provides the equivalent radius $\left[R^{*}=(a b)^{1 / 2}\right]$ and mean intensity of the isophote, its rms uncertainty, ellipticity, position angle, etc.

The isophotes were fitted using our $R$-band images (which were typically deeper than the $B$-band exposures). Then, we computed the mean flux and rms in the $B$ band and $\mathrm{H} \alpha$ images using the isophotes fitted in the $R$ band. In this way the colors were measured in exactly the same regions. We started the fitting procedure at the approximate position of the half-light radius, then we moved outward from that position; and finally, we moved inward using constant linear steps of between 1 and 5 pixels. The step size was determined by the depth and quality (spatial resolution) of the image.

In order to compute the error in the surface brightness and color profiles we start from the expression that relates the intensity in counts per pixel with the surface brightness,

$\mu_{\lambda}=C_{\lambda}-2.5 \log \left(I_{\lambda}-I_{\text {sky }, \lambda}\right)+5 \log \left(\operatorname{arcsec} \operatorname{pixel}^{-1}\right)$.

Thus, to a first approximation we can write the uncertainty in $\mu_{\lambda}$ as

$$
\Delta \mu_{\lambda}=\sqrt{\Delta C_{\lambda}^{2}+\left[\frac{2.5 \log (e)}{I_{\lambda}-I_{\mathrm{sky}, \lambda}} \Delta\left(I_{\lambda}-I_{\mathrm{sky}, \lambda}\right)\right]^{2}} .
$$

That can be expressed in terms of the rms uncertainty along the isophote and the error on the sky level as

$$
\Delta \mu_{\lambda}=\sqrt{\Delta C_{\lambda}^{2}+\left[\frac{2.5 \log (e)}{I_{\lambda}-I_{\text {sky }, \lambda}}\right]^{2}\left[\left(\frac{\mathrm{rms}_{\text {isophote }}}{\sqrt{N_{\text {isophote }}}}\right)^{2}+\Delta I_{\text {sky }, \lambda}^{2}\right]} .
$$

The term $\Delta I_{\text {sky, } \lambda}^{2}$ is actually composed of two terms: one is due to a combination of Poisson noise in the sky values and highfrequency (pixel-to-pixel) flat-fielding errors, and the other is due to low-frequency flat-fielding errors and the presence of reflections or gradients in the image background. This latter component may be dominant in the outermost part of the galaxy profiles as shown by Noeske et al. (2003). In order to determine these numbers we measured the value of the sky and its standard deviation in a total of 15-20 regions of $N_{\text {region }}$ pixels each around the position of the galaxy. If we now define $\left\langle\sigma_{\text {sky }}\right\rangle$ and $\left\langle\sigma_{\text {sky }}\right\rangle^{2}$ as the mean standard deviation and variance of the sky values measured in these individual regions, respectively, we obtain

$$
\Delta I_{\text {sky }, \lambda}^{2}=\frac{\left\langle\sigma_{\text {sky }}\right\rangle^{2}}{N_{\text {isophote }}}+\max \left(\sigma_{\langle\text {sky }\rangle}^{2}-\frac{\left\langle\sigma_{\text {sky }}\right\rangle^{2}}{N_{\text {region }}}, 0\right) .
$$

The second term of the sum can be neglected if the lowfrequency flat-fielding errors are negligible compared with the combined effect of the sky photon noise and the highfrequency flat-fielding errors.

In Figure 1 we plot the surface brightness and color profiles for each of the 114 galaxies in the original Atlas sample. The $1 \sigma$ error bars plotted combine in quadrature the standard deviation of the isophote mean and the error in the sky subtraction. The calibration errors for each of the bands are indicated by vertical bars in the lower left corner of the plot. Horizontal tick marks on the left vertical axis indicate the value of the HWHM of the PSF. Figure 1 shows that all the galaxies in the sample are clearly resolved, so these light profiles can be adequately used to study the distribution of the stellar populations in these galaxies. Even in the case of the very compact galaxy UM 404, which was observed with a very poor seeing (almost $4^{\prime \prime}$ in the $B$ band), its profile extends radially more than 3 times the PSF HWHM. The surface brightnesses and colors have been corrected for Galactic extinction but not for internal extinction. Despite the very low metallicity and, therefore, low expected dust content, internal extinction may be important in the innermost regions, where most of the current star formation activity is taking place (see 

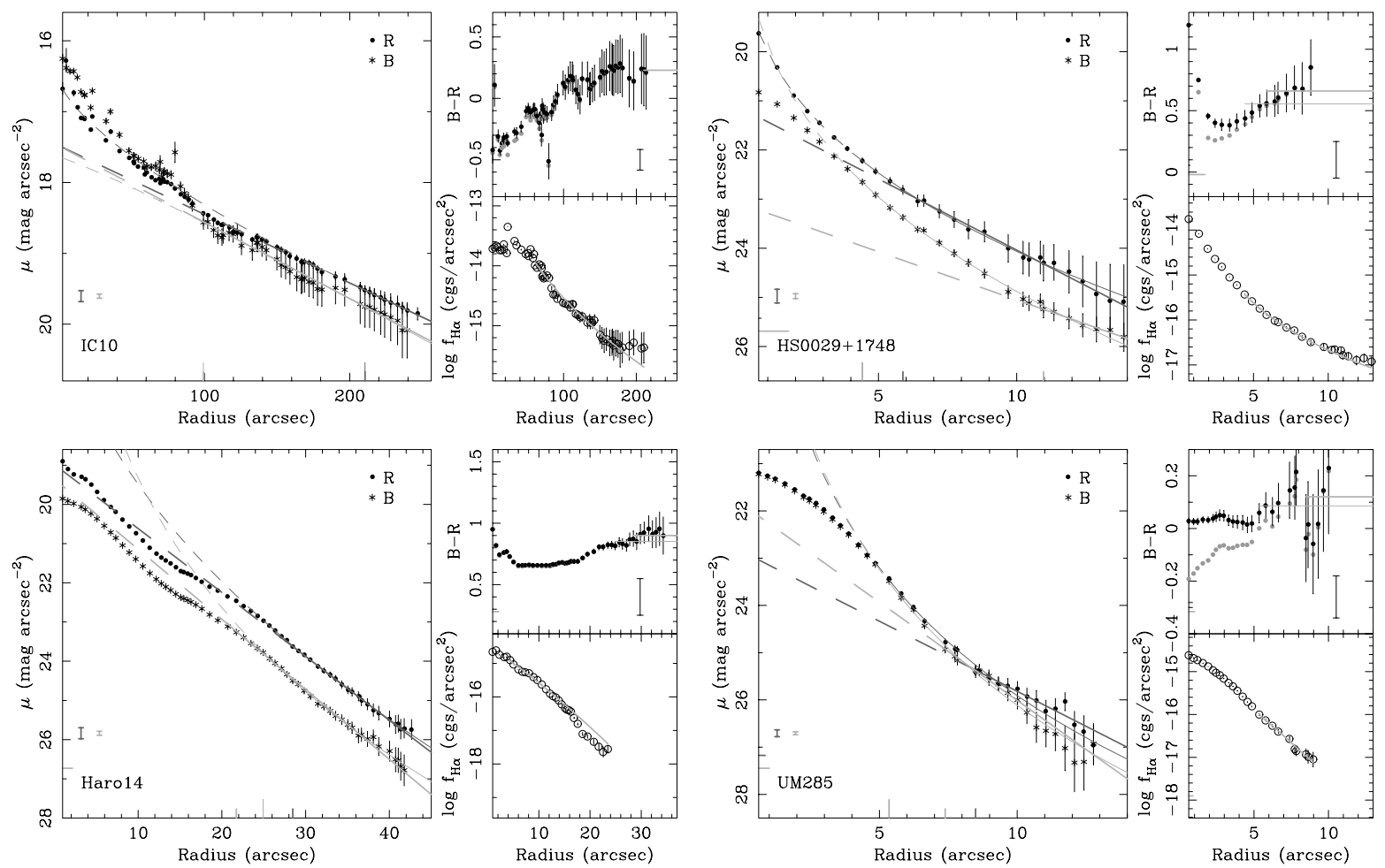

FIG. 1.-Surface brightness and color profiles of the galaxies in the BCD sample. Left panel: For each galaxy we plot the two surface brightness profiles (along with the $1 \sigma$ errors), one each for the $B$-band (asterisks) and $R$-band (filled circles) data. The error bars at the bottom left corner of the diagram show the error associated with the flux calibration of the $B$-band (light gray) and $R$-band (dark gray) images. We also plot the best-fitting exponential (thick solid lines) and Sérsic (thin solid lines) profiles of the USP (light gray for $B$ and dark gray lines for $R$ ), and the corresponding extrapolation to the center (dashed lines). The vertical ticks at the bottom of the plot indicate the position outward from which the surface brightness profile of the USP was fitted. In those galaxies where the inner radius plotted is smaller than the image HWHM, horizontal ticks at the left side of the plot show the extension of the $B$ (light gray) and $R$ bands (dark gray) PSF HWHM. Top right panel: We show the $(B-R)$ observed (black points) and ionized-gas contamination-corrected (gray points) color profiles with their corresponding $1 \sigma$ errors. The horizontal thick (thin) solid line marks the average $(B-R)$ color of the USP and the region where the best-fitting exponential (Sérsic) profile was derived. The error bar at the bottom right corner of this diagram shows the error in the $(B-R)$ color due to flux calibration uncertaintites. Bottom right panel: H $\alpha$ surface brightness profile in cgs units (ergs s $\mathrm{cm}^{-2}$ arcsec ${ }^{-2}$ ) and their corresponding $1 \sigma$ errors. The light gray solid line shows the best fitting Sersic profile. [See the electronic edition of the Supplement for a color version of this figure and additional panels.]

Noeske et al. 2003; Cannon et al. 2002). However, the effect of the dust on the structural properties and colors of the USP derived in this paper, which are measured at a considerable distance from the sites of current star formation, is probably negligible.

The surface brightness profiles obtained show a very high central surface brightness that decreases very rapidly with the galactocentric radius. For many of the galaxies in our sample a radius can be identified outside which the surface brightness starts to decrease at a much slower rate following an approximate exponential or de Vaucoleurs $r^{1 / 4}$ law, depending on the galaxy. We interpret this behavior as being due to the existence of two well-differentiated stellar populations, a young population that would be responsible for the high surface brightness (HSB) nuclear emission and a more evolved (fainter and redder) USP with a smooth, LSB profile dominating the outermost parts of the galaxy's surface brightness profile. This idea, which had been proposed in the past by different authors (see Papaderos et al. 1996a, 1996b and references therein), is supported by the fact than in many of the galaxies in our sample this radius coincides with a flattening in the color profile, also called the transition radius (Papaderos et al. 1996a, 1996b).

However, there are many cases where the analysis of the surface brightness profile alone does not allow identifying the radius from which the USP starts to dominate the galaxy's light distribution. This can be due to (1) the USP dominating the profile all the way to the center of the galaxy, (2) the relative con- tribution in luminosity of the two stellar populations changing gradually but in a very smooth way across the galaxy, (3) the transition taking place at a surface brightness very close or below our detection limit (i.e., the USP is undetected), or (4) the USP is not present, like it could the case of those galaxies suspect of forming stars for the first time (e.g., I Zw 18; Tol 65). In $\S 3.2$ we will describe the procedure used to determine (in a homogeneous way) the radius outside which the galaxy's surface brightness profile is dominated by the emission from its USP.

\subsection{Profile Fitting}

We did not find any simple function or combination of functions that adequately reproduces the surface brightness profile of the galaxies all the way from the nucleus to the outermost regions.

In order to compare the structural properties of the USP in our BCD sample with those observed in other dwarf galaxies, we fitted the surface brightness of the USP using both an exponential and a Sérsic law. The free parameters for this fitting procedure where those of the exponential and Sérsic laws plus the position of the radius from where the surface brightness profile is assumed to be dominated by the USP. In those cases where there was an obvious flattening of the color profile we only considered points external to the transition radius. The best-fitting set of parameters was obtained by minimizing the reduced $\chi^{2}$ (normalized to the degrees of freedom) of the fit. No fewer than five points were used for each fit. 
The position of the best-fitting innermost point of the fit (i.e., the radius outside which the USP dominates the profile) is shown in the Figure 1 by vertical tick marks at the bottom axis of the surface brightness plot. In most cases this radius is similar for both the exponential and Sérsic-law fits, which suggests the presence of a clear change in the surface brightness profile at this radius. In those cases where a significant difference is seen between the two fits, the best-fitting innermost point for the exponential law is usually placed at a larger galactocentric distance than the corresponding for the Sérsic law. In this plot we also show the resulting best-fitting exponential and Sérsic laws in the range of the fit (solid lines) and the corresponding extrapolation toward the galaxy center (dashed lines).

In Tables 1 and 2 the parameters of the best-fitting exponential and Sérsic laws are given. In Figures $2 a-2 f$ we show the parameters and $\chi_{r}^{2}$ from the fit of an exponential law to the $B$-band (Figs. $2 a-2 c$ ) and $R$-band (Figs. $2 d-2 f$ ) profiles. The distributions of best-fitting Sérsic indices for the $B$ and $R$ profiles are shown in Figures $2 g-2 h$, respectively.

Tables 1 and 2 show that the application of either of these laws to the broadband surface brightness profiles of the USP yields comparable low values of $\chi_{r}^{2}$. At very low values of $\chi_{r}^{2}$ $(<0.1)$ the exponential law seems to provide a slightly better fit, while at very high values of $\chi_{r}^{2}$ the Sérsic law is the one that more often yields a lower value for the reduced $\chi_{r}^{2}$. In this sense, for the 21 exponential fits with $\chi_{r}^{2}>1$ the $\chi_{r}^{2}$ of the corresponding Sérsic fit is better than that given by the exponential fit. However, this only represents $10 \%$ of the sample. For only five of the profiles is $\chi_{r}^{2}>3$.

In some cases although the best fit yielded relatively low $\chi_{r}^{2}$ values, inspection of the profile showed that fit was obtained using only a few points (never fewer than five) with their errors strongly dominated by the uncertainty in the background subtraction. In this situation, the errors of the different points used for the fit are largely correlated and the value of $\chi_{r}^{2}$ may be underestimated (see Tables 1 and 2). An example of this behavior is seen at the $B$-band profile of HS $0029+1748$.

The use of a Sérsic law has the advantage of encompassing the exponential law as a particular case. It also allows us to consider for BCD galaxies a relationship between the Sérsiclaw index and luminosity found for dwarf elliptical galaxies (see Graham \& Guzmán 2003). However, in the case of the USP of BCD galaxies, the region available to us for the fitting is relatively small and it is located at large distances from the galaxy center. Under these circumstances, the uncertainties and degeneracies between the parameters of the Sérsic law become extremely large and highly dependent on the particular region of the profile considered (Cairós et al. 2003; Noeske et al. 2003; see also $\S 4.2 .1$ ).

\section{RESULTS AND DISCUSSION}

\subsection{Surface Brightness and Color Profiles}

The surface brightness profiles shown in Figure 1 are typically characterized by an HSB component near the center of the galaxy superimposed on a nearly exponential LSB component associated with its USP. These characteristics in the profiles of BCD galaxies have been previously observed by various authors (Loose \& Thuan 1985; Papaderos et al. 1996a, 1996b; Cairós et al. 2001a, 2001b). Small departures of the surface brightness profile of the USP from an exponential law have been proposed. Doublier et al. $(1997,1999)$ argued that in approximately one-fourth of the BCD galaxies considered by them the profiles were better described by a de Vaucoleurs $r^{1 / 4}$ law. On the other hand, Noeske et al. (2003), using deep nearinfrared imaging, have recently proposed that a large fraction of BCD galaxies may have USP with surface brightness profiles showing a central flattening similar to the type $\mathrm{V}$ profiles found by Binggeli \& Cameron (1991) in some dwarf elliptical galaxies. In $\S 4.2$ we analyze in detail the morphology of the USP in our BCD sample of galaxies.

The color profiles obtained indicate that the HSB component commonly shows very blue colors, especially once the colors are corrected for line and nebular-continuum contamination. At larger radii, where the relative contribution of the HSB component becomes smaller, the colors tend to get redder. In most of the galaxies the color profile flattens at the radial position where the USP begins to dominate the galaxy's global surface brightness profile. This behavior was first observed by Papaderos et al. (1996a, 1996b) and more recently by Doublier et al. (1997, 1999) and Cairós et al. (2001a, 2001b). Papaderos et al. (1996a, $1996 \mathrm{~b})$ called the position where the color profile flattens the transition radius. Note that the much larger sample used in the present work compared with previous studies allows us to obtain, for the first time, statistically meaningful conclusions about the structural properties of BCDs as a class of objects. Of the 104 BCD galaxies in our sample, $70 \%$ (72 objects) show this kind of flattening (some examples are ISZ 399, Tol 2, Haro 2). About 17\% (18 galaxies) show a progressive reddening of the color profile in the outer parts of the galaxy. In some of the objects in this group the contamination from the HSB component may still be important at faintest surface brightness levels detected by our observations. Examples of these objects are NGC 4861 and Haro 9. Six objects (5\%) show a bluing of the color profile in the outermost parts of the galaxy. An example of this type of behavior is seen in the profile of Tol 1345-420. Finally, for a total of 9 galaxies the large errors in the outermost $(B-R)$ colors measured prevent us from determining the degree of flattening of the color profile beyond the transition radius.

\subsection{Underlying Stellar Population}

\subsubsection{Sérsic versus Exponential Law}

The Sérsic indices found by fitting both the $B$ - and $R$-band profiles suggest that the USP tends to have a surface brightness profile somewhat steeper than an exponential law. Moreover, about 40 of the galaxies show indices steeper than the de Vaucoleurs profile $(n=4)$. However, it is worth noting that (as we commented in $\S 3.2$ ) the values derived for the Sérsic-law parameters are highly uncertain and strongly dependent on the region considered for the fit. In particular, they strongly depend on the surface brightness of the few innermost points considered during the fit. At radial distances close to but beyond the position of the transition radius, some profiles show surface brightnesses in excess of what is expected from our best-fitting exponential law. Since these are the innermost points considered in our Sérsic-law fit, their value has a critical impact on the best-fitting index derived. We believe these intermediate radial distance regions may well be contaminated by the emission from the HSB component. This contamination would affect substantially the shape of the total surface brightness profile, but would have a smaller impact on the color profile. Some examples are NGC 1705, NGC 2915, Mrk 1423, NGC 3125, and ESO 572-G025.

In order to understand the effect of the particular region considered for the fit on the indices derived, we have also computed the best-fitting Sérsic index within the region used 
TABLE 1

Exponential-Law Fitting Parameters

\begin{tabular}{|c|c|c|c|c|c|c|c|}
\hline $\begin{array}{l}\text { Object Name } \\
\text { (1) }\end{array}$ & $\begin{array}{c}\alpha_{B} \\
(\operatorname{arcsec}) \\
(2)\end{array}$ & $\begin{array}{c}\mu_{B, 0} \\
\left(\mathrm{mag} \operatorname{arcsec}^{-2} \text { ) }\right. \\
(3)\end{array}$ & $\begin{array}{l}\chi_{r}^{2} \\
(4)\end{array}$ & $\begin{array}{c}\alpha_{R} \\
(\operatorname{arcsec}) \\
(5)\end{array}$ & $\begin{array}{c}\mu_{R, 0} \\
\left(\operatorname{mag} \operatorname{arcsec}^{-2}\right) \\
(6)\end{array}$ & $\begin{array}{l}\chi_{r}^{2} \\
(7)\end{array}$ & $\begin{array}{c}\text { Notes } \\
(8)\end{array}$ \\
\hline IC 10 & $100.8 \pm 3.8$ & $17.48 \pm 0.06$ & 0.17 & $112.1 \pm 1.8$ & $17.48 \pm 0.09$ & 0.00 & \\
\hline HS $0029+1748 \ldots \ldots \ldots \ldots \ldots . . . .$. & $5.5 \pm 0.4$ & $23.09 \pm 0.18$ & 0.02 & $3.8 \pm 0.2$ & $21.14 \pm 0.17$ & 0.06 & a \\
\hline Haro 14 & $6.1 \pm 0.1$ & $19.36 \pm 0.10$ & 0.49 & $6.7 \pm 0.1$ & $18.98 \pm 0.15$ & 0.05 & \\
\hline 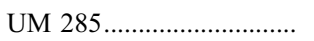 & $2.6 \pm 0.2$ & $21.86 \pm 0.24$ & 0.32 & $3.7 \pm 0.4$ & $22.85 \pm 0.29$ & 0.36 & a \\
\hline UCM 0049-0045 ............ & $8.7 \pm 0.7$ & $22.46 \pm 0.16$ & 0.81 & $10.6 \pm 0.4$ & $21.53 \pm 0.27$ & 0.09 & \\
\hline 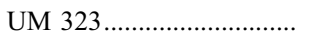 & $2.3 \pm 0.1$ & $19.58 \pm 0.17$ & 0.30 & $2.4 \pm 0.1$ & $19.11 \pm 0.14$ & 0.86 & \\
\hline Mrk 996 & $4.2 \pm 0.1$ & $20.56 \pm 0.08$ & 0.30 & $4.5 \pm 0.1$ & $19.75 \pm 0.15$ & 1.06 & \\
\hline 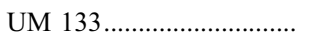 & $9.9 \pm 0.4$ & $23.03 \pm 0.13$ & 0.29 & $12.0 \pm 0.6$ & $22.37 \pm 0.18$ & 0.15 & \\
\hline 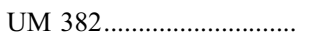 & $1.8 \pm 0.1$ & $21.45 \pm 0.08$ & 0.45 & $1.9 \pm 0.1$ & $21.42 \pm 0.27$ & 0.26 & \\
\hline$\ldots$ & $2.1 \pm 0.1$ & $21.91 \pm 0.15$ & 0.68 & $2.1 \pm 0.2$ & $22.46 \pm 0.30$ & 0.51 & \\
\hline KUG 0207-016A.............. & $6.3 \pm 0.2$ & $21.49 \pm 0.12$ & 0.37 & $7.1 \pm 0.2$ & $21.39 \pm 0.14$ & 0.11 & \\
\hline 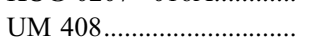 & $1.4 \pm 0.1$ & $20.23 \pm 0.22$ & 0.38 & $1.6 \pm 0.1$ & $19.63 \pm 0.13$ & 0.92 & \\
\hline 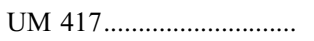 & $1.5 \pm 0.1$ & $20.24 \pm 0.20$ & 0.05 & $2.0 \pm 0.1$ & $21.14 \pm 0.15$ & 0.20 & \\
\hline Mrk 370 & $12.4 \pm 0.2$ & $21.38 \pm 0.04$ & 0.16 & $13.1 \pm 0.1$ & $20.19 \pm 0.12$ & 0.68 & \\
\hline Mrk 600 & $4.6 \pm 0.1$ & $20.78 \pm 0.06$ & 0.07 & $4.8 \pm 0.1$ & $20.59 \pm 0.16$ & 0.26 & \\
\hline 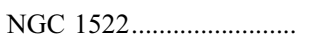 & $9.8 \pm 0.1$ & $21.78 \pm 0.02$ & 0.29 & $9.6 \pm 0.1$ & $20.56 \pm 0.06$ & 0.57 & \\
\hline 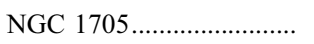 & $17.8 \pm 0.1$ & $22.33 \pm 0.03$ & 0.05 & $17.4 \pm 0.1$ & $21.09 \pm 0.06$ & 0.05 & \\
\hline II Zw 33 & $4.4 \pm 0.1$ & $19.61 \pm 0.05$ & 1.69 & $5.0 \pm 0.2$ & $19.25 \pm 0.16$ & 2.54 & \\
\hline 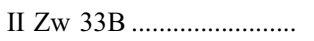 & $6.5 \pm 0.6$ & $22.98 \pm 0.21$ & 0.19 & $9.1 \pm 2.0$ & $22.48 \pm 0.50$ & 0.14 & \\
\hline II Zw 40 & $15.2 \pm 3.9$ & $19.76 \pm 0.62$ & 0.01 & $15.5 \pm 0.6$ & $18.97 \pm 0.10$ & 6.28 & \\
\hline 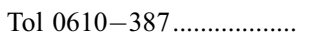 & $6.7 \pm 0.2$ & $22.91 \pm 0.10$ & 0.23 & $7.4 \pm 0.1$ & $21.96 \pm 0.06$ & 0.03 & \\
\hline Mrk 5 & $4.3 \pm 0.2$ & $20.59 \pm 0.11$ & 0.37 & $5.2 \pm 0.1$ & $20.24 \pm 0.13$ & 0.29 & \\
\hline Mrk 7 & $4.5 \pm 0.1$ & $19.41 \pm 0.04$ & 1.48 & $5.0 \pm 0.1$ & $19.00 \pm 0.19$ & 3.33 & \\
\hline Mrk 86 & $14.4 \pm 0.4$ & $20.57 \pm 0.09$ & 0.06 & $21.8 \pm 0.4$ & $20.88 \pm 0.12$ & 0.01 & \\
\hline 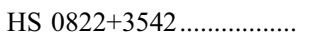 & $1.9 \pm 0.1$ & $21.20 \pm 0.07$ & 0.35 & $1.8 \pm 0.1$ & $21.50 \pm 0.17$ & 0.35 & \\
\hline 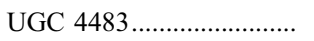 & $14.9 \pm 0.9$ & $23.12 \pm 0.14$ & 0.19 & $16.1 \pm 1.1$ & $22.33 \pm 0.18$ & 0.33 & a \\
\hline 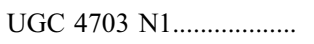 & $6.1 \pm 0.3$ & $23.07 \pm 0.13$ & 0.25 & $5.9 \pm 0.3$ & $22.21 \pm 0.16$ & 0.25 & \\
\hline UGC 4703 N2..................... & $4.6 \pm 0.2$ & $22.92 \pm 0.14$ & 0.40 & $4.3 \pm 0.2$ & $21.87 \pm 0.17$ & 0.65 & \\
\hline Mrk 1416 & $2.6 \pm 0.1$ & $20.49 \pm 0.07$ & 0.50 & $3.0 \pm 0.1$ & $20.28 \pm 0.10$ & 0.96 & \\
\hline Mrk 108 & $7.5 \pm 0.3$ & $22.10 \pm 0.09$ & 0.53 & $8.3 \pm 0.4$ & $21.64 \pm 0.11$ & 0.13 & a \\
\hline Mrk 400 & $8.9 \pm 0.5$ & $22.31 \pm 0.17$ & 0.90 & $12.2 \pm 0.6$ & $22.35 \pm 0.16$ & 0.25 & \\
\hline 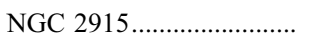 & $45.0 \pm 2.0$ & $23.11 \pm 0.10$ & 0.01 & $39.1 \pm 5.4$ & $21.48 \pm 0.36$ & 0.01 & a \\
\hline I Zw 18 & $4.2 \pm 0.2$ & $22.60 \pm 0.16$ & 0.19 & $7.0 \pm 1.0$ & $24.00 \pm 0.28$ & 0.36 & $\mathrm{~b}$ \\
\hline Mrk 1418 & $12.6 \pm 0.3$ & $22.79 \pm 0.09$ & 0.21 & $13.7 \pm 0.3$ & $21.35 \pm 0.20$ & 0.11 & \\
\hline 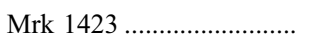 & $18.9 \pm 8.2$ & $24.00 \pm 1.28$ & 0.01 & $22.2 \pm 1.1$ & $22.97 \pm 0.16$ & 0.04 & a \\
\hline 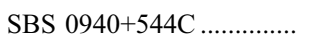 & $2.8 \pm 0.1$ & $21.15 \pm 0.08$ & 0.28 & $3.2 \pm 0.1$ & $21.40 \pm 0.11$ & 0.21 & \\
\hline Mrk 709 & $2.4 \pm 0.1$ & $20.75 \pm 0.12$ & 0.10 & $2.4 \pm 0.1$ & $19.86 \pm 0.10$ & 0.32 & \\
\hline Mrk 1426 & $4.0 \pm 0.1$ & $21.65 \pm 0.11$ & 1.26 & $5.8 \pm 0.2$ & $22.36 \pm 0.16$ & 0.25 & a \\
\hline 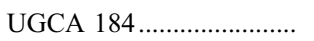 & $7.0 \pm 0.3$ & $23.08 \pm 0.11$ & 0.16 & $7.5 \pm 0.2$ & $22.95 \pm 0.13$ & 0.11 & \\
\hline Mrk 409 & $5.0 \pm 0.2$ & $19.75 \pm 0.16$ & 0.46 & $5.1 \pm 0.1$ & $18.84 \pm 0.13$ & 0.64 & \\
\hline 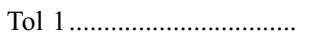 & $2.8 \pm 0.1$ & $20.30 \pm 0.11$ & 2.08 & $3.0 \pm 0.1$ & $20.17 \pm 0.11$ & 1.01 & \\
\hline Tol 2 & $12.9 \pm 0.2$ & $22.91 \pm 0.05$ & 0.09 & $13.3 \pm 0.2$ & $21.95 \pm 0.07$ & 0.09 & \\
\hline 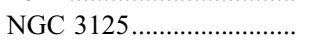 & $15.2 \pm 0.6$ & $22.92 \pm 0.13$ & 0.02 & $15.7 \pm 0.4$ & $21.86 \pm 0.11$ & 0.01 & a \\
\hline 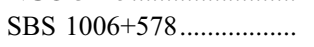 & $2.9 \pm 0.1$ & $20.99 \pm 0.12$ & 0.88 & $3.0 \pm 0.1$ & $20.32 \pm 0.14$ & 0.44 & \\
\hline Haro 2 & $15.4 \pm 0.2$ & $22.69 \pm 0.06$ & 0.29 & $16.7 \pm 0.3$ & $22.02 \pm 0.11$ & 0.47 & \\
\hline Mrk 1434 & $2.1 \pm 0.2$ & $21.20 \pm 0.24$ & 0.82 & $2.6 \pm 0.2$ & $21.31 \pm 0.25$ & 0.80 & a \\
\hline Haro 3 & $9.5 \pm 0.2$ & $21.11 \pm 0.08$ & 0.22 & $9.8 \pm 0.2$ & $20.10 \pm 0.14$ & 0.13 & \\
\hline SBS $1054+504 \ldots \ldots \ldots \ldots \ldots$ & $3.8 \pm 0.1$ & $21.56 \pm 0.12$ & 0.28 & $4.0 \pm 0.1$ & $20.93 \pm 0.17$ & 0.17 & \\
\hline Haro 4 & $4.0 \pm 0.4$ & $21.42 \pm 0.28$ & 0.16 & $3.8 \pm 0.2$ & $20.87 \pm 0.22$ & 0.07 & a \\
\hline 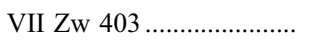 & $25.8 \pm 2.4$ & $23.75 \pm 0.17$ & 0.08 & $24.6 \pm 1.1$ & $22.88 \pm 0.14$ & 0.01 & a \\
\hline Mrk 178 & $14.1 \pm 0.3$ & $22.71 \pm 0.07$ & 0.14 & $15.0 \pm 0.2$ & $21.99 \pm 0.10$ & 0.16 & \\
\hline 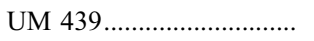 & $9.9 \pm 0.4$ & $22.60 \pm 0.11$ & 0.33 & $9.3 \pm 0.3$ & $21.62 \pm 0.10$ & 1.62 & \\
\hline Mrk 1450 & $9.0 \pm 1.9$ & $24.39 \pm 0.39$ & 0.40 & $8.6 \pm 1.4$ & $23.25 \pm 0.37$ & 0.05 & $\mathrm{~b}$ \\
\hline 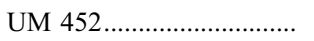 & $8.0 \pm 0.4$ & $22.58 \pm 0.15$ & 0.20 & $8.1 \pm 0.2$ & $21.30 \pm 0.14$ & 0.16 & \\
\hline SBS $1147+520 \ldots \ldots \ldots \ldots \ldots \ldots . .$. & $4.9 \pm 0.6$ & $24.22 \pm 0.34$ & 0.02 & $5.1 \pm 0.6$ & $22.65 \pm 0.28$ & 0.11 & a \\
\hline Tol 17 & $4.9 \pm 0.2$ & $22.08 \pm 0.11$ & 0.37 & $5.1 \pm 0.1$ & $21.02 \pm 0.09$ & 0.34 & \\
\hline 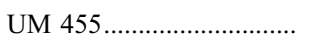 & $3.9 \pm 0.4$ & $23.54 \pm 0.30$ & 0.41 & $3.8 \pm 0.2$ & $22.23 \pm 0.14$ & 0.32 & a \\
\hline 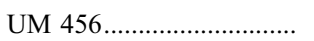 & $4.8 \pm 0.1$ & $20.71 \pm 0.12$ & 0.13 & $6.1 \pm 0.2$ & $20.75 \pm 0.07$ & 1.10 & \\
\hline 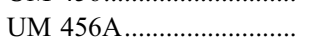 & $3.0 \pm 0.2$ & $21.57 \pm 0.25$ & 0.50 & $5.4 \pm 0.5$ & $22.90 \pm 0.26$ & 0.23 & $\mathrm{~b}$ \\
\hline Pox 4 & $3.0 \pm 0.1$ & $19.73 \pm 0.19$ & 0.54 & $3.3 \pm 0.1$ & $19.81 \pm 0.16$ & 0.30 & \\
\hline ES O572-G025 .................. & $13.6 \pm 0.2$ & $23.84 \pm 0.05$ & 0.22 & $13.8 \pm 0.2$ & $22.80 \pm 0.05$ & 0.17 & \\
\hline VCC 0001 & $3.1 \pm 0.1$ & $20.12 \pm 0.05$ & 0.16 & $3.1 \pm 0.1$ & $18.99 \pm 0.04$ & 0.27 & \\
\hline Mrk 1313 & $3.1 \pm 0.2$ & $21.36 \pm 0.24$ & 0.22 & $3.3 \pm 0.1$ & $20.92 \pm 0.15$ & 0.24 & a \\
\hline
\end{tabular}


TABLE 1 -Continued

\begin{tabular}{|c|c|c|c|c|c|c|c|}
\hline $\begin{array}{c}\text { Object Name } \\
\text { (1) }\end{array}$ & $\begin{array}{c}\alpha_{B} \\
(\operatorname{arcsec}) \\
(2)\end{array}$ & $\begin{array}{c}\mu_{B, 0} \\
\left(\operatorname{mag~arcsec}^{-2} \text { ) }\right. \\
\text { (3) }\end{array}$ & $\begin{array}{l}\chi_{r}^{2} \\
(4)\end{array}$ & $\begin{array}{c}\alpha_{R} \\
(\operatorname{arcsec}) \\
(5)\end{array}$ & $\begin{array}{c}\mu_{R, 0} \\
\text { (mag arcsec } \\
\text { (6) }\end{array}$ & $\begin{array}{l}\chi_{r}^{2} \\
(7)\end{array}$ & $\begin{array}{c}\text { Notes } \\
(8)\end{array}$ \\
\hline 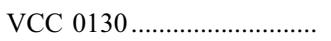 & $2.5 \pm 0.1$ & $21.00 \pm 0.06$ & 0.92 & $2.5 \pm 0.1$ & $20.21 \pm 0.03$ & 0.94 & \\
\hline Haro 8 & $8.7 \pm 0.2$ & $21.23 \pm 0.06$ & 1.09 & $9.5 \pm 0.3$ & $20.33 \pm 0.12$ & 2.45 & \\
\hline 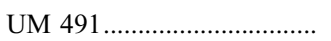 & $3.9 \pm 0.3$ & $21.52 \pm 0.26$ & 0.27 & $4.1 \pm 0.2$ & $20.83 \pm 0.16$ & 0.40 & \\
\hline ISZ 399 & $8.8 \pm 0.1$ & $21.85 \pm 0.02$ & 0.32 & $8.7 \pm 0.1$ & $20.50 \pm 0.06$ & 0.48 & \\
\hline VCC 0459 & $4.6 \pm 0.1$ & $20.95 \pm 0.10$ & 0.61 & $4.8 \pm 0.1$ & $20.03 \pm 0.09$ & 1.32 & \\
\hline VCC 0655 & $14.1 \pm 0.2$ & $21.45 \pm 0.06$ & 0.47 & $14.0 \pm 0.2$ & $20.14 \pm 0.04$ & 0.60 & \\
\hline Tol 65 & $1.8 \pm 0.1$ & $21.09 \pm 0.21$ & 5.61 & $2.0 \pm 0.1$ & $20.83 \pm 0.17$ & 5.64 & \\
\hline VCC 0848 & $10.4 \pm 0.2$ & $22.54 \pm 0.06$ & 0.35 & $11.4 \pm 0.2$ & $21.75 \pm 0.03$ & 0.30 & \\
\hline Mrk 209 & $25.1 \pm 1.0$ & $24.12 \pm 0.07$ & 0.13 & $27.4 \pm 2.9$ & $23.66 \pm 0.24$ & 0.04 & \\
\hline Mrk 1329 & $10.9 \pm 0.3$ & $21.70 \pm 0.06$ & 0.63 & $11.2 \pm 0.2$ & $20.84 \pm 0.10$ & 2.91 & \\
\hline 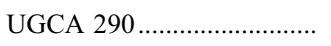 & $4.4 \pm 0.1$ & $20.59 \pm 0.05$ & 0.08 & $5.3 \pm 0.1$ & $20.49 \pm 0.14$ & 0.10 & \\
\hline VCC 1750 & $3.8 \pm 0.1$ & $22.40 \pm 0.09$ & 0.26 & $4.0 \pm 0.1$ & $21.45 \pm 0.07$ & 0.67 & \\
\hline 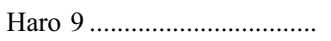 & $9.5 \pm 0.1$ & $20.52 \pm 0.05$ & 0.25 & $10.2 \pm 0.1$ & $19.75 \pm 0.10$ & 0.07 & \\
\hline 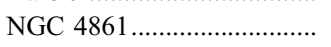 & $21.1 \pm 0.2$ & $21.51 \pm 0.04$ & 0.61 & $24.2 \pm 0.2$ & $20.95 \pm 0.09$ & 0.71 & \\
\hline UM 533 & $13.8 \pm 0.4$ & $22.75 \pm 0.08$ & 0.24 & $14.2 \pm 0.2$ & $21.69 \pm 0.11$ & 0.12 & \\
\hline Mrk 450 & $14.1 \pm 0.4$ & $22.95 \pm 0.09$ & 0.07 & $15.7 \pm 0.3$ & $22.29 \pm 0.11$ & 0.05 & \\
\hline NGC 5058 & $8.3 \pm 0.2$ & $21.18 \pm 0.10$ & 0.37 & $8.8 \pm 0.2$ & $20.59 \pm 0.09$ & 0.47 & \\
\hline 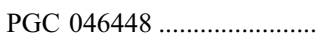 & $4.4 \pm 0.1$ & $21.82 \pm 0.12$ & 0.30 & $4.9 \pm 0.2$ & $21.00 \pm 0.15$ & 1.35 & a \\
\hline Pox 186 & $1.7 \pm 0.1$ & $22.24 \pm 0.17$ & 0.05 & $2.1 \pm 0.3$ & $22.31 \pm 0.37$ & 0.08 & \\
\hline 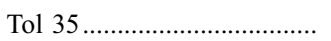 & $4.5 \pm 0.1$ & $19.44 \pm 0.08$ & 0.29 & $4.6 \pm 0.1$ & $18.58 \pm 0.06$ & 0.34 & \\
\hline 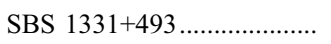 & $5.4 \pm 0.1$ & $20.42 \pm 0.07$ & 0.74 & $6.1 \pm 0.1$ & $20.14 \pm 0.18$ & 0.02 & \\
\hline Tol 85 & $2.2 \pm 0.1$ & $19.69 \pm 0.19$ & 0.54 & $2.7 \pm 0.1$ & $20.17 \pm 0.19$ & 0.59 & \\
\hline 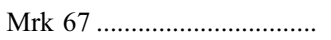 & $7.3 \pm 0.4$ & $23.76 \pm 0.14$ & 0.52 & $9.4 \pm 0.6$ & $23.08 \pm 0.16$ & 0.44 & \\
\hline Mrk 1480 & $4.1 \pm 0.3$ & $22.61 \pm 0.21$ & 0.40 & $3.9 \pm 0.2$ & $21.45 \pm 0.20$ & 0.27 & a \\
\hline 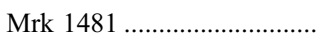 & $12.0 \pm 0.6$ & $24.40 \pm 0.09$ & 0.34 & $12.5 \pm 0.4$ & $23.48 \pm 0.08$ & 0.06 & \\
\hline Tol $1345-420 \ldots \ldots \ldots \ldots$ & $4.2 \pm 0.5$ & $21.90 \pm 0.26$ & 2.23 & $5.5 \pm 0.4$ & $21.98 \pm 0.19$ & 0.22 & $\mathrm{~b}$ \\
\hline 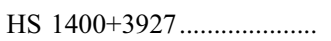 & $2.6 \pm 0.1$ & $21.83 \pm 0.09$ & 0.23 & $2.4 \pm 0.1$ & $20.39 \pm 0.12$ & 0.38 & \\
\hline SBS $1415+437 \ldots \ldots \ldots \ldots$ & $4.7 \pm 0.1$ & $21.02 \pm 0.08$ & 1.02 & $5.1 \pm 0.1$ & $20.52 \pm 0.14$ & 0.12 & \\
\hline 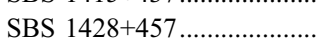 & $4.1 \pm 0.2$ & $21.42 \pm 0.24$ & 0.62 & $4.4 \pm 0.3$ & $20.67 \pm 0.30$ & 3.19 & \\
\hline Tol 1434+032 & $5.7 \pm 0.2$ & $22.06 \pm 0.09$ & 0.37 & $6.6 \pm 0.1$ & $21.98 \pm 0.08$ & 0.08 & \\
\hline Mrk 475 & $3.6 \pm 0.1$ & $21.70 \pm 0.09$ & 0.28 & $4.5 \pm 0.1$ & $21.49 \pm 0.11$ & 0.25 & \\
\hline 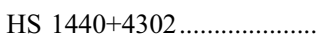 & $3.3 \pm 0.2$ & $23.06 \pm 0.15$ & 0.43 & $3.5 \pm 0.1$ & $21.87 \pm 0.14$ & 0.32 & \\
\hline 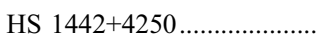 & $4.4 \pm 0.1$ & $20.63 \pm 0.07$ & 0.16 & $4.8 \pm 0.1$ & $20.26 \pm 0.08$ & 0.26 & \\
\hline 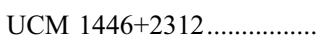 & $4.8 \pm 0.2$ & $21.19 \pm 0.11$ & 1.56 & $5.4 \pm 0.2$ & $20.35 \pm 0.13$ & 3.28 & \\
\hline Tol 1448+116 ........................ & $2.7 \pm 0.3$ & $21.49 \pm 0.31$ & 0.20 & $3.2 \pm 0.2$ & $21.02 \pm 0.17$ & 1.22 & a \\
\hline II Zw 70 & $10.1 \pm 0.8$ & $23.59 \pm 0.22$ & 0.37 & $8.8 \pm 0.3$ & $22.25 \pm 0.17$ & 0.11 & a \\
\hline II Zw 71 & $6.8 \pm 0.1$ & $20.70 \pm 0.16$ & 0.04 & $7.9 \pm 0.1$ & $20.26 \pm 0.12$ & 0.09 & \\
\hline I Zw 115 & $6.3 \pm 0.1$ & $21.02 \pm 0.10$ & 0.34 & $6.9 \pm 0.1$ & $20.27 \pm 0.09$ & 0.24 & \\
\hline SBS $1533+574 \ldots \ldots \ldots \ldots$ & $2.8 \pm 0.2$ & $21.23 \pm 0.20$ & 0.22 & $3.2 \pm 0.1$ & $20.55 \pm 0.13$ & 0.29 & \\
\hline I Zw 123 & $3.9 \pm 0.2$ & $21.70 \pm 0.19$ & 0.40 & $4.5 \pm 0.1$ & $21.13 \pm 0.13$ & 0.55 & \\
\hline HS $1609+4827 \ldots \ldots \ldots \ldots \ldots$ & $2.7 \pm 0.1$ & $19.66 \pm 0.15$ & 1.23 & $2.9 \pm 0.1$ & $19.00 \pm 0.12$ & 7.98 & \\
\hline UCM 1612+1308 ................... & $1.1 \pm 0.2$ & $20.14 \pm 0.64$ & 1.81 & $1.4 \pm 0.1$ & $20.37 \pm 0.10$ & 0.09 & \\
\hline UGCA $412 \ldots \ldots \ldots \ldots \ldots$ & $5.6 \pm 0.2$ & $22.55 \pm 0.13$ & 0.24 & $5.5 \pm 0.3$ & $21.25 \pm 0.17$ & 0.48 & \\
\hline 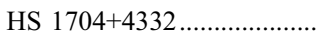 & $1.3 \pm 0.1$ & $21.34 \pm 0.35$ & 0.65 & $1.6 \pm 0.1$ & $21.86 \pm 0.24$ & 0.02 & \\
\hline NGC $6789 \ldots \ldots$ & $12.9 \pm 0.1$ & $21.49 \pm 0.04$ & 0.11 & $13.4 \pm 0.2$ & $20.49 \pm 0.08$ & 0.25 & \\
\hline Tol 1924-416 ......................... & $9.3 \pm 0.7$ & $23.04 \pm 0.24$ & 0.05 & $6.6 \pm 0.2$ & $20.79 \pm 0.12$ & 0.40 & $\mathrm{a}$ \\
\hline 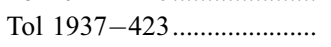 & $8.6 \pm 1.7$ & $23.63 \pm 0.58$ & 0.01 & $6.1 \pm 0.4$ & $21.30 \pm 0.22$ & 0.11 & $\mathrm{~b}$ \\
\hline Mrk 900 & $9.2 \pm 0.2$ & $21.74 \pm 0.06$ & 0.09 & $9.1 \pm 0.3$ & $20.76 \pm 0.14$ & 0.58 & \\
\hline Mrk 314 & $12.0 \pm 0.4$ & $22.11 \pm 0.09$ & 0.08 & $12.2 \pm 0.7$ & $21.48 \pm 0.27$ & 0.01 & $\mathrm{a}$ \\
\hline Mrk 324 & $3.6 \pm 0.2$ & $20.92 \pm 0.20$ & 0.93 & $3.4 \pm 0.1$ & $19.97 \pm 0.21$ & 0.27 & \\
\hline Mrk 328 & $4.7 \pm 0.1$ & $21.21 \pm 0.12$ & 0.02 & $4.6 \pm 0.1$ & $20.19 \pm 0.12$ & 0.27 & \\
\hline
\end{tabular}

Notes.-Columns stand for (1) galaxy name; (2) scale length of the best-fitting exponential profile of the USP in the $B$ band (arcsec); (3) extrapolated central surface brightness of the USP in the $B$ band $\left(\operatorname{mag} \operatorname{arcsec}^{-2}\right)$; (4) reduced $\chi^{2}$ of the best exponential fit in the $B$ band; (5-7) same as (2-4) for the $R$ band; (8) notes. Table 1 is also available in machine-readable form in the electronic edition of the Astrophysical Journal Supplement.

${ }^{a}$ Errors in the points used for the fit are correlated. $\chi_{r}^{2}$ could be underestimated.

$\mathrm{b}$ Errors strongly correlated. The value of $\chi_{r}^{2}$ should be considered a lower limit (see text for details).

for the fit to an exponential law. Figures $2 g-2 i$ show the comparison between the distributions of Sérsic indices obtained in this way and those obtained using different regions for the Sérsic and exponential-law fits. Although the number of objects with profiles steeper than exponential is still significant, the number of objects with Sérsic indices $n>4$ is significantly lower when the fit is performed within the same region used for the exponential fit.

The large differences obtained by the two methods demonstrate the strong dependence of the best-fitting Sérsic-law 
TABLE 2

Sérsic-Law Fitting Parameters

\begin{tabular}{|c|c|c|c|c|c|c|c|c|c|c|c|c|c|}
\hline $\begin{array}{l}\text { Object Name } \\
\text { (1) }\end{array}$ & $\begin{array}{c}1 / n_{B} \\
(2)\end{array}$ & $\begin{array}{c}r_{B, 0} \\
(\operatorname{arcsec}) \\
\quad(3)\end{array}$ & $\mu_{B, 0}\left(\operatorname{mag} \operatorname{arcsec}^{-2}\right)$ & $\begin{array}{l}\chi_{r}^{2} \\
(5)\end{array}$ & $\begin{array}{l}1 / n_{R} \\
(6)\end{array}$ & $\begin{array}{c}r_{R, 0} \\
(\operatorname{arcsec}) \\
\quad(7)\end{array}$ & $\begin{array}{c}\mu_{R, 0} \\
\left(\mathrm{mag} \operatorname{arcsec}^{-2} \text { ) }\right. \\
(8)\end{array}$ & $\begin{array}{l}\chi_{r}^{2} \\
(9)\end{array}$ & $\begin{array}{l}1 / n_{\mathrm{H} \alpha} \\
(10)\end{array}$ & $\begin{array}{l}r_{\mathrm{H} \alpha, 0} \\
(\operatorname{arcsec}) \\
(11)\end{array}$ & $\begin{array}{c}\log f_{\mathrm{H} \alpha, 0} \\
\left(\operatorname{cgs} \operatorname{arcsec}^{-2}\right) \\
(12)\end{array}$ & $\begin{array}{c}\chi_{r}^{2} \\
(13)\end{array}$ & $\begin{array}{c}\text { Notes } \\
(14)\end{array}$ \\
\hline IC $10 \ldots$. & $1.11 \pm 0.53$ & $115 \pm 60$ & $17.64 \pm 5.67$ & 0.17 & $0.70 \pm 1.43$ & $50 \pm 100$ & $16.55^{\mathrm{a}}$ & 0.00 & $0.41 \pm 0.10$ & $7.02 \pm 8.95$ & $-11.31 \pm 1.01$ & 5.97 & \\
\hline HS $0029+1748 \ldots \ldots \ldots$ & $0.33 \pm 0.12$ & $0.01 \pm 0.15$ & $15.48 \pm 7.66$ & 0.21 & $0.37 \pm 0.29$ & $0.06 \pm 1.02$ & $17.01^{\mathrm{a}}$ & 0.05 & $0.05 \pm 0.01$ & $<0.01$ & & 1.29 & \\
\hline Haro $14 \ldots \ldots \ldots \ldots \ldots$ & $0.18 \pm 0.22$ & $<0.01$ & & 0.13 & $0.50 \pm 0.23$ & $0.33 \pm 1.63$ & $13.48^{\mathrm{a}}$ & 0.07 & $1.19 \pm 0.01$ & $10.45 \pm 0.04$ & $-14.55^{\mathrm{a}}$ & $>100$ & $\mathrm{~b}$ \\
\hline 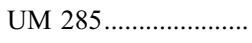 & $0.05 \pm 0.05$ & $<0.01$ & $\ldots$ & 0.56 & $0.05 \pm 0.00$ & $<0.01$ & $\ldots$ & 0.77 & $1.14 \pm 0.69$ & $4.28 \pm 2.55$ & $-14.60^{\mathrm{a}}$ & 0.32 & \\
\hline UCM $0049-0045 \ldots$. & $0.05 \pm 0.00$ & $<0.01$ & $\ldots$ & 0.59 & $0.06 \pm 0.03$ & $<0.01$ & $\ldots$ & 0.40 & $2.91 \pm 0.21$ & $9.95 \pm 0.26$ & $-14.83 \pm 0.08$ & 0.21 & \\
\hline UM $323 \ldots \ldots \ldots \ldots \ldots \ldots$ & $0.45 \pm 0.27$ & $0.07 \pm 0.63$ & $14.06^{\mathrm{a}}$ & 0.12 & $0.52 \pm 0.19$ & $0.21 \pm 0.49$ & $15.45 \pm 8.78$ & 0.23 & $0.05 \pm 0.17$ & $<0.01$ & $\ldots$ & 0.80 & \\
\hline Mrk $996 \ldots \ldots \ldots \ldots \ldots$ & $0.67 \pm 0.11$ & $1.31 \pm 0.76$ & $18.91 \pm 0.89$ & 0.17 & $0.66 \pm 0.05$ & $1.36 \pm 0.38$ & $18.12 \pm 0.38$ & 0.35 & $0.05 \pm 0.00$ & $<0.01$ & & 1.22 & \\
\hline 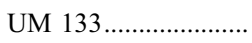 & $0.56 \pm 0.66$ & $1.66 \pm 8.06$ & $20.84^{\mathrm{a}}$ & 0.28 & $0.05 \pm 0.41$ & $<0.01$ & & 0.13 & $0.60 \pm 0.08$ & $4.02 \pm 1.25$ & $-14.03 \pm 0.24$ & 2.71 & \\
\hline UM $382 \ldots \ldots \ldots \ldots \ldots$ & $1.19 \pm 0.18$ & $2.35 \pm 0.47$ & $21.78 \pm 0.30$ & 0.38 & $0.25 \pm 0.69$ & $<0.01$ & $11.16^{\mathrm{a}}$ & 0.18 & $0.05 \pm 0.00$ & $<0.01$ & & 2.10 & $\mathrm{~b}$ \\
\hline UM $404 \ldots \ldots \ldots \ldots \ldots \ldots$ & $0.32 \pm 0.55$ & $<0.01$ & $16.15^{\mathrm{a}}$ & 0.58 & $0.05 \pm 0.75$ & $<0.01$ & & 0.40 & $0.19 \pm 0.05$ & $<0.01$ & & 6.37 & \\
\hline KUG 0207-016A..... & $0.30 \pm 0.24$ & $<0.01$ & $12.19^{\mathrm{a}}$ & 0.17 & $0.72 \pm 0.39$ & $2.84 \pm 4.37$ & $20.02^{\mathrm{a}}$ & 0.10 & $0.05 \pm 0.10$ & $<0.01$ & & 1.16 & $\mathrm{~b}$ \\
\hline 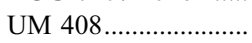 & $0.27 \pm 0.63$ & $<0.01$ & $11.82^{\mathrm{a}}$ & 0.31 & $1.10 \pm 0.20$ & $1.85 \pm 0.57$ & $19.89 \pm 0.60$ & 0.88 & $0.38 \pm 0.21$ & $0.23 \pm 0.83$ & $-12.50 \pm 5.71$ & 0.57 & $\mathrm{~b}$ \\
\hline 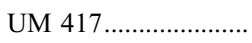 & $2.60 \pm 0.46$ & $5.13 \pm 0.57$ & $22.97 \pm 0.27$ & 0.12 & $1.17 \pm 0.42$ & $2.60 \pm 1.32$ & $21.58 \pm 3.42$ & 0.18 & $1.78 \pm 0.06$ & $5.23 \pm 0.08$ & $-15.02 \pm 0.03$ & 1.61 & $\mathrm{~b}$ \\
\hline Mrk $370 \ldots \ldots \ldots \ldots \ldots$ & $1.05 \pm 0.16$ & $13.50 \pm 3.40$ & $21.50 \pm 0.39$ & 0.16 & $0.81 \pm 0.04$ & $8.34 \pm 0.94$ & $19.63 \pm 0.18$ & 0.35 & $0.05 \pm 0.00$ & $<0.01$ & $\ldots$ & 20.30 & \\
\hline Mrk $600 \ldots \ldots \ldots \ldots \ldots \ldots$ & $0.82 \pm 0.26$ & $2.90 \pm 2.04$ & $20.11 \pm 3.66$ & 0.07 & $0.71 \pm 0.19$ & $2.04 \pm 1.51$ & $19.49 \pm 1.68$ & 0.16 & $0.05 \pm 0.08$ & $<0.01$ & & 1.26 & \\
\hline 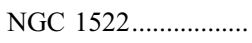 & $1.19 \pm 0.02$ & $13.64 \pm 0.37$ & $22.31 \pm 0.04$ & 0.34 & $1.31 \pm 0.02$ & $15.87 \pm 0.22$ & $21.45 \pm 0.06$ & 0.44 & $0.32 \pm 0.10$ & $0.02 \pm 0.15$ & $\ldots$ & 1.10 & \\
\hline 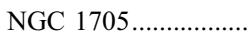 & $0.51 \pm 0.11$ & $1.35 \pm 1.80$ & $18.48 \pm 2.21$ & 0.05 & $0.50 \pm 0.02$ & $1.26 \pm 0.28$ & $17.35 \pm 0.25$ & 0.24 & $0.22 \pm 0.12$ & $0.02 \pm 1.82$ & $\ldots$ & 0.30 & \\
\hline 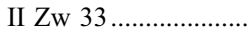 & $0.91 \pm 0.04$ & $3.60 \pm 0.32$ & $19.35 \pm 0.12$ & 1.49 & $0.68 \pm 0.02$ & $1.59 \pm 0.14$ & $17.55 \pm 0.14$ & 2.83 & $0.05 \pm 0.00$ & $<0.01$ & $\ldots$ & 1.50 & \\
\hline 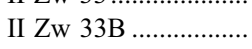 & $0.05 \pm 0.32$ & $<0.01$ & $\ldots$ & 0.21 & $0.05 \pm 0.88$ & $<0.01$ & $\ldots$ & 0.23 & $1.35 \pm 1.04$ & $8.14 \pm 5.25$ & $-14.93^{\mathrm{a}}$ & 0.52 & \\
\hline 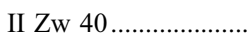 & $0.06 \pm 1.47$ & $<0.01$ & $\ldots$ & 0.01 & $0.11 \pm 0.07$ & $<0.01$ & $\ldots$ & 3.34 & $0.11 \pm 0.00$ & $<0.01$ & $\ldots$ & $>100$ & $\mathrm{c}$ \\
\hline Tol $0610-387 \ldots \ldots \ldots . .$. & $0.31 \pm 0.10$ & $0.01 \pm 0.16$ & $16.07 \pm 6.63$ & 0.20 & $0.84 \pm 0.22$ & $4.91 \pm 2.88$ & $21.38 \pm 1.24$ & 0.06 & $0.05 \pm 0.00$ & $<0.01$ & $\ldots$ & 4.41 & \\
\hline Mrk 5 & $0.59 \pm 0.20$ & $0.80 \pm 1.17$ & $18.29 \pm 6.57$ & 0.26 & $0.72 \pm 0.11$ & $2.28 \pm 1.08$ & $19.16 \pm 0.72$ & 0.14 & $0.71 \pm 0.03$ & $3.55 \pm 0.30$ & $-13.70 \pm 0.07$ & 4.61 & \\
\hline Mrk 7 ......................... & $0.95 \pm 0.03$ & $4.00 \pm 0.27$ & $19.24 \pm 0.10$ & 1.37 & $0.67 \pm 0.03$ & $1.59 \pm 0.27$ & $17.40 \pm 0.27$ & 2.02 & $0.05 \pm 0.00$ & $<0.01$ & $\ldots$ & 2.32 & \\
\hline Mrk 86 & $0.88 \pm 0.82$ & $10.6 \pm 15.4$ & $20.11^{\mathrm{a}}$ & 0.06 & $0.15 \pm 0.95$ & $<0.01$ & & 0.01 & $0.05 \pm 0.00$ & $<0.01$ & & 1.24 & \\
\hline HS $0822+3542 \ldots \ldots \ldots$ & $1.10 \pm 0.22$ & $2.25 \pm 0.73$ & $21.43 \pm 0.61$ & 0.32 & $0.65 \pm 0.20$ & $0.55 \pm 0.53$ & $19.95 \pm 3.59$ & 0.28 & $0.35 \pm 0.30$ & $0.10 \pm 1.18$ & $-12.02^{\mathrm{a}}$ & 0.83 & \\
\hline 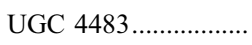 & $0.36 \pm 1.24$ & $0.24 \pm 15.76$ & $19.13^{\mathrm{a}}$ & 0.19 & $1.67 \pm 1.12$ & $28.01 \pm 14.55$ & $23.12^{\mathrm{a}}$ & 0.32 & $0.05 \pm 0.99$ & $<0.01$ & $\ldots$ & 0.05 & $\mathrm{c}$ \\
\hline UGC $4703 \mathrm{~N} 1 \ldots \ldots \ldots . . . .$. & $0.05 \pm 0.51$ & $<0.01$ & & 0.18 & $0.05 \pm 0.11$ & $<0.01$ & $\ldots$ & 0.43 & $0.05 \pm 0.00$ & $<0.01$ & $\ldots$ & 3.15 & \\
\hline UGC 4703 N2 .............. & $0.18 \pm 0.52$ & $<0.01$ & $10.03^{\mathrm{a}}$ & 0.32 & $0.15 \pm 0.45$ & $<0.01$ & $\ldots$ & 0.55 & $\ldots$ & $\ldots$ & $\ldots$ & $\ldots$ & \\
\hline Mrk 1416 & $0.93 \pm 0.10$ & $2.19 \pm 0.54$ & $20.24 \pm 0.39$ & 0.48 & $0.69 \pm 0.10$ & $1.11 \pm 0.55$ & $18.98 \pm 0.67$ & 0.50 & $0.59 \pm 0.07$ & $1.46 \pm 0.52$ & $-13.06 \pm 0.31$ & 1.23 & \\
\hline Mrk 108 & $0.05 \pm 0.01$ & $<0.01$ & $\ldots$ & 0.98 & $0.05 \pm 0.04$ & $<0.01$ & $\ldots$ & 0.34 & $0.05 \pm 0.00$ & $<0.01$ & $\ldots$ & $>100$ & \\
\hline 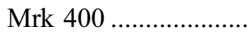 & $0.05 \pm 0.08$ & $<0.01$ & $\ldots$ & 0.61 & $0.05 \pm 0.18$ & $<0.01$ & $\ldots$ & 0.21 & $0.05 \pm 0.00$ & $<0.01$ & $\ldots$ & 1.29 & \\
\hline 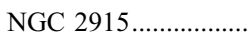 & $0.19 \pm 0.11$ & $<0.01$ & $10.46^{\mathrm{a}}$ & 0.05 & $0.05 \pm 0.04$ & $<0.01$ & $\ldots$ & 0.08 & $0.07 \pm 0.01$ & $<0.01$ & $\ldots$ & 32.40 & \\
\hline I Zw 18 & $3.00 \pm 1.14$ & $13.29 \pm 5.28$ & $24.92^{\mathrm{a}}$ & 0.10 & $3.00 \pm 1.33$ & $13.28 \pm 0.01$ & $24.83^{\mathrm{a}}$ & 0.07 & $0.05 \pm 0.00$ & $<0.01$ & $\ldots$ & 5.85 & $\mathrm{c}$ \\
\hline Mrk 1418 & $0.13 \pm 0.04$ & $<0.01$ & $\ldots$ & 0.23 & $0.13 \pm 0.04$ & $<0.01$ & $\ldots$ & 0.18 & $0.05 \pm 0.00$ & $<0.01$ & $\ldots$ & 7.83 & \\
\hline Mrk $1423 \ldots \ldots \ldots \ldots \ldots \ldots$ & $0.53 \pm 0.03$ & $1.82 \pm 0.41$ & $20.64 \pm 0.20$ & 0.54 & $0.05 \pm 0.04$ & $<0.01$ & $\ldots$ & 0.15 & $0.05 \pm 0.00$ & $<0.01$ & $\ldots$ & 2.40 & \\
\hline SBS $0940+544 C \ldots \ldots$ & $1.29 \pm 0.10$ & $4.53 \pm 0.46$ & $22.00 \pm 0.17$ & 0.47 & $1.06 \pm 0.14$ & $3.53 \pm 0.76$ & $21.53 \pm 0.33$ & 0.20 & $1.52 \pm 0.05$ & $7.24 \pm 0.14$ & $-14.93 \pm 0.03$ & 6.46 & \\
\hline Mrk 709 & $0.20 \pm 0.15$ & $<0.01$ & $\ldots$ & 0.11 & $0.34 \pm 0.13$ & $<0.01$ & $12.68 \pm 9.70$ & 0.19 & $2.18 \pm 1.00$ & $8.28 \pm 3.33$ & $-16.32 \pm 2.92$ & 0.38 & \\
\hline Mrk $1426 \ldots \ldots \ldots \ldots . . .$. & $0.05 \pm 0.06$ & $<0.01$ & $\ldots$ & 0.47 & $0.05 \pm 0.03$ & $<0.01$ & $\ldots$ & 0.54 & $0.05 \pm 0.03$ & $<0.01$ & $\ldots$ & 0.55 & \\
\hline UGCA $184 \ldots \ldots \ldots \ldots . . . . . . . .$. & $0.05 \pm 0.11$ & $<0.01$ & & 0.14 & $0.05 \pm 0.13$ & $<0.01$ & $\ldots$ & 0.19 & $3.00 \pm 1.17$ & $16.73 \pm 6.71$ & $-16.53^{\mathrm{a}}$ & 0.35 & \\
\hline Mrk 409 ......................... & $0.77 \pm 0.27$ & $2.25 \pm 2.49$ & $18.27 \pm 7.20$ & 0.42 & $0.05 \pm 0.15$ & $<0.01$ & $\ldots$ & 0.19 & $0.05 \pm 1.41$ & $<0.01$ & $\ldots$ & 0.12 & $\mathrm{~b}$ \\
\hline 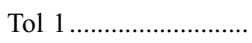 & $0.17 \pm 0.13$ & $<0.01$ & & 0.26 & $0.57 \pm 0.11$ & $0.40 \pm 0.40$ & $17.03 \pm 1.67$ & 0.22 & $0.80 \pm 0.06$ & $3.40 \pm 0.37$ & $-14.13 \pm 0.09$ & 3.89 & \\
\hline Tol 2 & $0.93 \pm 0.48$ & $11.14 \pm 8.88$ & $22.71^{\mathrm{a}}$ & 0.09 & $1.14 \pm 0.32$ & $16.60 \pm 6.76$ & $22.28 \pm 2.07$ & 0.09 & $0.05 \pm 0.00$ & $<0.01$ & $\ldots$ & 31.00 & \\
\hline 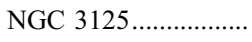 & $0.32 \pm 0.06$ & $0.02 \pm 0.10$ & $13.02 \pm 2.66$ & 0.11 & $0.28 \pm 0.07$ & $<0.01$ & $10.68 \pm 4.42$ & 0.07 & $0.85 \pm 0.18$ & $22.08 \pm 6.86$ & $-14.78 \pm 0.38$ & 0.29 & \\
\hline SBS $1006+578 \ldots \ldots \ldots$ & $0.74 \pm 0.09$ & $1.26 \pm 0.48$ & $19.78 \pm 0.55$ & 1.25 & $0.78 \pm 0.12$ & $1.57 \pm 0.66$ & $19.39 \pm 0.66$ & 0.59 & $0.77 \pm 0.66$ & $3.52 \pm 4.11$ & $-13.85^{\mathrm{a}}$ & 0.92 & \\
\hline Haro 2 & $0.18 \pm 0.05$ & $<0.01$ & $\ldots$ & 0.25 & $0.12 \pm 0.04$ & $<0.01$ & $\ldots$ & 0.25 & $0.09 \pm 0.16$ & $<0.01$ & $\ldots$ & 0.02 & \\
\hline
\end{tabular}


TABLE 2-Continued

\begin{tabular}{|c|c|c|c|c|c|c|c|c|c|c|c|c|c|}
\hline $\begin{array}{l}\text { Object Name } \\
\text { (1) }\end{array}$ & $\begin{array}{c}1 / n_{B} \\
(2)\end{array}$ & $\begin{array}{c}r_{B, 0} \\
(\operatorname{arcsec}) \\
(3)\end{array}$ & $\mu_{B, 0}\left(\operatorname{mag} \operatorname{arcsec}^{-2}\right)$ & $\begin{array}{l}\chi_{r}^{2} \\
(5)\end{array}$ & $\begin{array}{c}1 / n_{R} \\
(6)\end{array}$ & $\begin{array}{c}r_{R, 0} \\
(\operatorname{arcsec}) \\
\quad(7)\end{array}$ & $\begin{array}{c}\mu_{R, 0} \\
\left(\operatorname{mag~arcsec}^{-2} \text { ) }\right. \\
(8)\end{array}$ & $\begin{array}{l}\chi_{r}^{2} \\
(9)\end{array}$ & $\begin{array}{c}1 / n_{\mathrm{H} \alpha} \\
(10)\end{array}$ & $\begin{array}{c}r_{\mathrm{H} \alpha, 0} \\
(\operatorname{arcsec}) \\
(11)\end{array}$ & $\begin{array}{c}\log f_{\mathrm{H} \alpha, 0} \\
\left(\operatorname{cgs} \operatorname{arcsec}^{-2}\right) \\
(12)\end{array}$ & $\begin{array}{c}\chi_{r}^{2} \\
(13)\end{array}$ & $\begin{array}{c}\text { Notes } \\
(14)\end{array}$ \\
\hline Mrk 1434 & $0.46 \pm 0.28$ & $0.09 \pm 0.66$ & $16.95^{\mathrm{a}}$ & 0.46 & $0.11 \pm 0.19$ & $<0.01$ & & 0.21 & $0.17 \pm 0.05$ & $<0.01$ & 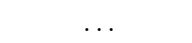 & 1.66 & $\mathrm{~b}$ \\
\hline Haro 3 & $0.34 \pm 0.09$ & $0.03 \pm 0.19$ & $12.70 \pm 4.64$ & 0.13 & $0.06 \pm 0.29$ & $<0.01$ & $\ldots$ & 0.02 & $0.05 \pm 0.12$ & $<0.01$ & $\ldots$ & 0.56 & b \\
\hline SBS $1054+504 \ldots \ldots \ldots \ldots$ & $0.51 \pm 0.07$ & $0.33 \pm 0.24$ & $18.23 \pm 0.86$ & 0.37 & $0.53 \pm 0.05$ & $0.42 \pm 0.18$ & $17.85 \pm 0.50$ & 0.11 & $0.05 \pm 0.27$ & $<0.01$ & & 0.56 & \\
\hline Haro 4 & $0.05 \pm 0.36$ & $<0.01$ & $\ldots$ & 0.20 & $1.01 \pm 1.34$ & $3.91 \pm 5.94$ & $20.91^{\mathrm{a}}$ & 0.07 & $0.42 \pm 0.01$ & $0.36 \pm 0.05$ & $-11.28 \pm 0.09$ & 31.97 & $\mathrm{~b}$ \\
\hline VII Zw $403 \ldots \ldots \ldots \ldots \ldots$ & $0.05 \pm 0.18$ & $<0.01$ & $\ldots$ & 0.16 & $0.05 \pm 0.17$ & $<0.01$ & & 0.03 & $0.05 \pm 0.00$ & $<0.01$ & $\ldots$ & 0.52 & \\
\hline Mrk 178 & $1.29 \pm 0.29$ & $21.84 \pm 6.88$ & $23.41 \pm 2.08$ & 0.13 & $1.02 \pm 0.17$ & $15.61 \pm 5.05$ & $22.05 \pm 0.62$ & 0.16 & $0.05 \pm 0.00$ & $<0.01$ & $\ldots$ & 0.82 & \\
\hline UM $439 \ldots$ & $0.05 \pm 0.11$ & $<0.01$ & $\ldots$ & 0.40 & $0.39 \pm 0.12$ & $0.22 \pm 0.73$ & $17.66 \pm 4.14$ & 1.32 & $0.05 \pm 0.13$ & $<0.01$ & $\ldots$ & 0.43 & \\
\hline Mrk 1450 . & $0.05 \pm 0.00$ & $<0.01$ & $\ldots$ & 1.39 & $0.05 \pm 0.00$ & $<0.01$ & & 1.42 & $0.05 \pm 0.00$ & $<0.01$ & & 5.30 & $\mathrm{~b}$ \\
\hline UM 452 . & $0.05 \pm 0.16$ & $<0.01$ & $\ldots$ & 0.21 & $0.08 \pm 0.20$ & $<0.01$ & & 0.13 & $3.00 \pm 0.01$ & $6.48 \pm 0.02$ & $-15.19^{\mathrm{a}}$ & 30.03 & \\
\hline SBS $1147+520 \ldots$ & $0.05 \pm 1.43$ & $<0.01$ & $\ldots$ & 0.02 & $0.05 \pm 1.14$ & $<0.01$ & & 0.08 & $3.00 \pm 0.32$ & $4.57 \pm 0.18$ & $-15.52 \pm 0.08$ & 0.21 & $\mathrm{~b}$ \\
\hline Tol $17 \ldots \ldots$ & $0.56 \pm 0.08$ & $0.71 \pm 0.44$ & $19.42 \pm 0.83$ & 0.36 & $0.63 \pm 0.06$ & $1.27 \pm 0.46$ & $19.08 \pm 0.43$ & 0.38 & $0.29 \pm 0.15$ & $0.01 \pm 0.26$ & $\ldots$ & 1.01 & \\
\hline 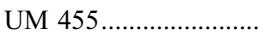 & $0.05 \pm 0.41$ & $<0.01$ & $\ldots$ & 0.45 & $1.17 \pm 0.97$ & $4.99 \pm 4.06$ & $22.64^{\mathrm{a}}$ & 0.32 & $0.84 \pm 0.01$ & $1.74 \pm 0.03$ & $-13.62 \pm 0.01$ & 2.77 & b \\
\hline UM 456.............................. & $1.75 \pm 1.02$ & $13.08 \pm 7.56$ & $22.94^{\mathrm{a}}$ & 0.08 & $1.87 \pm 0.23$ & $14.30 \pm 1.43$ & $22.25 \pm 0.22$ & 0.42 & $0.05 \pm 0.09$ & $<0.01$ & $\ldots$ & 0.21 & \\
\hline UM 456A & $0.05 \pm 0.03$ & $<0.01$ & $\ldots$ & 0.35 & $0.05 \pm 0.01$ & $<0.01$ & & 0.73 & $2.77 \pm 0.31$ & $8.73 \pm 0.13$ & $-15.46 \pm 0.05$ & 0.22 & \\
\hline Pox 4 & $0.05 \pm 0.09$ & $<0.01$ & $\ldots$ & 0.78 & $1.11 \pm 0.47$ & $4.26 \pm 3.11$ & $20.31^{\mathrm{a}}$ & 0.30 & $0.05 \pm 0.01$ & $<0.01$ & $\ldots$ & 0.71 & $\mathrm{~b}$ \\
\hline ESO $572-\mathrm{G} 025 \ldots \ldots \ldots . .$. & $0.26 \pm 0.01$ & $<0.01$ & $15.56 \pm 0.45$ & 0.90 & $0.05 \pm 0.00$ & $<0.01$ & & 0.48 & $0.05 \pm 0.00$ & $<0.01$ & . & 1.13 & \\
\hline 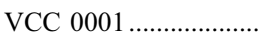 & $0.90 \pm 0.18$ & $2.45 \pm 1.11$ & $19.78 \pm 0.84$ & 0.14 & $0.54 \pm 0.31$ & $0.37 \pm 1.26$ & $16.00^{\mathrm{a}}$ & 0.02 & $0.87 \pm 0.10$ & $2.70 \pm 0.40$ & $-14.88 \pm 0.14$ & 1.33 & \\
\hline Mrk 1313 & $1.62 \pm 1.01$ & $6.70 \pm 3.97$ & $22.77^{\mathrm{a}}$ & 0.20 & $1.44 \pm 0.84$ & $6.06 \pm 3.60$ & $21.98^{\mathrm{a}}$ & 0.22 & $0.56 \pm 0.14$ & $1.47 \pm 1.11$ & $-13.56 \pm 1.20$ & 1.93 & $\mathrm{~b}$ \\
\hline VCC $0130 \ldots \ldots \ldots \ldots \ldots$ & $1.01 \pm 0.04$ & $2.55 \pm 0.17$ & $21.03 \pm 0.10$ & 0.92 & $0.95 \pm 0.03$ & $2.28 \pm 0.17$ & $20.06 \pm 0.10$ & 0.79 & $0.05 \pm 0.30$ & $<0.01$ & & 0.84 & \\
\hline Haro 8 ............................. & $0.87 \pm 0.06$ & $6.44 \pm 1.01$ & $20.84 \pm 0.21$ & 0.95 & $0.60 \pm 0.04$ & $2.24 \pm 0.55$ & $18.62 \pm 0.28$ & 0.80 & $1.34 \pm 0.02$ & $9.35 \pm 0.07$ & $-14.37 \pm 0.01$ & 7.51 & \\
\hline 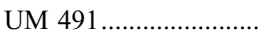 & $0.22 \pm 0.53$ & $<0.01$ & $\ldots$ & 0.21 & $0.19 \pm 0.33$ & $<0.01$ & & 0.16 & $0.59 \pm 0.08$ & $1.27 \pm 0.55$ & $-13.24 \pm 0.42$ & 1.85 & \\
\hline ISZ 399 & $0.89 \pm 0.03$ & $6.87 \pm 0.60$ & $21.50 \pm 0.12$ & 0.23 & $1.01 \pm 0.02$ & $8.88 \pm 0.41$ & $20.52 \pm 0.09$ & 0.47 & $0.75 \pm 0.01$ & $2.03 \pm 0.04$ & $-13.00 \pm 0.01$ & 19.16 & \\
\hline VCC $0459 \ldots . .$. & $0.71 \pm 0.11$ & $1.70 \pm 0.90$ & $41 \pm 0.91$ & 0.45 & $0.59 \pm 0.08$ & $0.84 \pm 0.52$ & $17.50 \pm 0.87$ & 0.69 & $0.05 \pm 0.00$ & $<0.01$ & $\ldots$ & 3.86 & \\
\hline VCC $0655 \ldots \ldots \ldots \ldots \ldots$ & $0.62 \pm 0.02$ & $3.36 \pm 0.44$ & $19.52 \pm 0.15$ & 0.52 & $0.77 \pm 0.04$ & $7.18 \pm 1.16$ & $19.18 \pm 0.22$ & 0.20 & $0.05 \pm 0.00$ & $<0.01$ & $\ldots$ & 1.58 & \\
\hline Tol 65 & $1.17 \pm 0.06$ & $2.41 \pm 0.21$ & $21.49 \pm 0.14$ & 5.21 & $1.02 \pm 0.05$ & $2.03 \pm 0.20$ & $20.88 \pm 0.14$ & 5.63 & $0.07 \pm 0.04$ & $<0.01$ & $\ldots$ & 6.93 & \\
\hline VCC $0848 \ldots \ldots \ldots \ldots \ldots . . . . .$. & $0.91 \pm 0.15$ & $8.67 \pm 2.86$ & $22.31 \pm 0.49$ & 0.33 & $0.83 \pm 0.13$ & $7.78 \pm 2.57$ & $21.29 \pm 0.43$ & 0.28 & $0.05 \pm 0.01$ & $<0.01$ & $\ldots$ & 0.59 & \\
\hline Mrk 209 & $0.05 \pm 0.26$ & $<0.01$ & $\ldots$ & 0.11 & $0.05 \pm 0.01$ & $<0.01$ & & 0.22 & $0.05 \pm 0.25$ & $<0.01$ & $\ldots$ & 1.09 & \\
\hline Mrk 1329 & $0.46 \pm 0.04$ & $0.70 \pm 0.38$ & $18.63 \pm 0.50$ & 0.68 & $0.72 \pm 0.02$ & $5.04 \pm 0.51$ & $19.86 \pm 0.14$ & 1.97 & $0.05 \pm 0.01$ & $<0.01$ & $\ldots$ & 5.47 & \\
\hline UGCA $290 \ldots \ldots \ldots \ldots$ & $0.84 \pm 0.21$ & $2.84 \pm 1.82$ & $19.87 \pm 1.54$ & 0.08 & $0.69 \pm 0.40$ & $1.90 \pm 3.06$ & $19.08^{\mathrm{a}}$ & 0.07 & $3.00 \pm 0.01$ & $27.36 \pm 0.79$ & $-16.31 \pm 0.02$ & 1.81 & \\
\hline VCC $1750 \ldots \ldots \ldots \ldots \ldots \ldots$ & $1.00 \pm 0.35$ & $3.79 \pm 2.19$ & $22.40 \pm 4.09$ & 0.26 & $1.25 \pm 0.17$ & $5.94 \pm 1.13$ & $22.09 \pm 0.33$ & 0.46 & $0.61 \pm 0.04$ & $0.96 \pm 0.20$ & $-13.13 \pm 0.17$ & 1.77 & \\
\hline Haro $9 \ldots \ldots \ldots \ldots \ldots$ & $0.95 \pm 0.04$ & $8.48 \pm 0.83$ & $20.35 \pm 0.15$ & 0.27 & $0.99 \pm 0.05$ & $9.99 \pm 1.02$ & $19.73 \pm 0.17$ & 0.07 & $0.09 \pm 0.01$ & $<0.01$ & $\ldots$ & 9.48 & \\
\hline NGC 4861........................ & $0.86 \pm 0.03$ & $14.79 \pm 1.48$ & $20.98 \pm 0.14$ & 0.52 & $0.91 \pm 0.02$ & $20.30 \pm 0.98$ & $20.73 \pm 0.10$ & 0.60 & $0.05 \pm 0.00$ & $<0.01$ & $\ldots$ & 4.95 & \\
\hline UM $533 \ldots \ldots \ldots \ldots \ldots \ldots \ldots$ & $0.61 \pm 0.19$ & $3.14 \pm 3.48$ & $20.80 \pm 3.83$ & 0.31 & $0.62 \pm 0.18$ & $3.71 \pm 3.55$ & $20.04 \pm 2.78$ & 0.06 & $0.85 \pm 0.01$ & $5.12 \pm 0.09$ & $-13.86 \pm 0.01$ & 3.08 & \\
\hline Mrk 450 & $0.12 \pm 0.11$ & $<0.01$ & $\ldots$ & 0.22 & $0.05 \pm 0.05$ & $<0.01$ & & 0.12 & $0.05 \pm 0.01$ & $<0.01$ & $\ldots$ & 0.88 & \\
\hline NGC 5058...................... & $0.64 \pm 0.08$ & $2.24 \pm 1.01$ & $19.43 \pm 0.60$ & 1.06 & $0.61 \pm 0.10$ & $1.99 \pm 1.34$ & $18.62 \pm 0.90$ & 0.37 & $0.05 \pm 0.61$ & $<0.01$ & $\ldots$ & 1.54 & \\
\hline PGC $046448 \ldots \ldots \ldots \ldots \ldots . . . . .$. & $0.36 \pm 0.10$ & $0.02 \pm 0.12$ & $14.57 \pm 4.66$ & 0.28 & $0.05 \pm 0.04$ & $<0.01$ & & 0.99 & $0.60 \pm 0.01$ & $1.00 \pm 0.03$ & $-13.26 \pm 0.02$ & 65.51 & \\
\hline Pox $186 \ldots \ldots \ldots \ldots \ldots \ldots \ldots$ & $0.12 \pm 0.42$ & $<0.01$ & $\ldots$ & 0.24 & $0.35 \pm 0.28$ & $0.02 \pm 0.56$ & $17.92^{\mathrm{a}}$ & 0.45 & $0.05 \pm 0.00$ & $<0.01$ & $\ldots$ & 2.23 & $\mathrm{~b}$ \\
\hline Tol 35 & $1.96 \pm 0.07$ & $13.17 \pm 0.36$ & $21.79 \pm 0.07$ & 0.66 & $0.87 \pm 0.10$ & $3.14 \pm 1.02$ & $17.83 \pm 0.63$ & 0.39 & $1.68 \pm 0.09$ & $18.91 \pm 0.33$ & $-15.09 \pm 0.03$ & 1.04 & \\
\hline SBS $1331+493 \ldots \ldots \ldots \ldots$ & $1.34 \pm 0.08$ & $8.68 \pm 0.69$ & $21.19 \pm 0.14$ & 0.46 & $1.23 \pm 0.07$ & $8.24 \pm 0.63$ & $20.38 \pm 0.20$ & 0.54 & $0.99 \pm 0.02$ & $9.47 \pm 0.27$ & $-14.29 \pm 0.03$ & 28.48 & \\
\hline Tol $85 \ldots \ldots \ldots \ldots \ldots \ldots$ & $0.68 \pm 0.36$ & $0.56 \pm 1.26$ & $16.97^{\mathrm{a}}$ & 0.50 & $0.05 \pm 0.24$ & $<0.01$ & $\ldots$ & 0.24 & $0.05 \pm 0.09$ & $<0.01$ & $\ldots$ & 0.87 & $\mathrm{~b}$ \\
\hline Mrk $67 .$. & $0.05 \pm 0.17$ & $<0.01$ & $\ldots$ & 0.26 & $0.05 \pm 0.00$ & $<0.01$ & & 0.62 & $0.05 \pm 0.00$ & $<0.01$ & $\ldots$ & 1.81 & \\
\hline Mrk 1480 & $0.13 \pm 0.09$ & $<0.01$ & $\ldots$ & 0.36 & $0.30 \pm 0.11$ & $<0.01$ & $12.57^{\mathrm{a}}$ & 0.19 & $0.05 \pm 0.00$ & $<0.01$ & $\ldots$ & 0.85 & \\
\hline Mrk 1481 & $0.05 \pm 0.10$ & $<0.01$ & $\ldots$ & 0.35 & $0.06 \pm 0.29$ & $<0.01$ & & 0.05 & $1.20 \pm 0.08$ & $7.44 \pm 0.34$ & $-15.17 \pm 0.06$ & 25.29 & $\mathrm{~b}$ \\
\hline Tol $1345-420 \ldots \ldots \ldots \ldots . . .$. & $0.05 \pm 0.02$ & $<0.01$ & $\ldots$ & 0.41 & $0.05 \pm 0.05$ & $<0.01$ & & 0.24 & $0.05 \pm 0.00$ & $<0.01$ & $\ldots$ & 23.37 & \\
\hline HS $1400+3927 \ldots \ldots \ldots \ldots$ & $0.54 \pm 0.16$ & $0.37 \pm 0.47$ & $19.45 \pm 3.55$ & 0.34 & $0.46 \pm 0.09$ & $0.15 \pm 0.18$ & $17.22 \pm 1.19$ & 1.06 & $0.15 \pm 0.36$ & $<0.01$ & $\cdots$ & 0.09 & \\
\hline SBS $1415+437 \ldots \ldots \ldots \ldots$ & $1.16 \pm 0.14$ & $6.12 \pm 1.19$ & $21.42 \pm 0.32$ & 0.98 & $0.34 \pm 0.40$ & $0.03 \pm 2.38$ & $14.77^{\mathrm{a}}$ & 0.04 & $0.19 \pm 0.03$ & $<0.01$ & $\ldots$ & 9.76 & \\
\hline
\end{tabular}


TABLE 2-Continued

\begin{tabular}{|c|c|c|c|c|c|c|c|c|c|c|c|c|c|}
\hline $\begin{array}{l}\text { Object Name } \\
\text { (1) }\end{array}$ & $\begin{array}{c}1 / n_{B} \\
(2)\end{array}$ & $\begin{array}{c}r_{B, 0} \\
(\operatorname{arcsec}) \\
(3)\end{array}$ & $\mu_{B, 0}\left(\operatorname{mag} \operatorname{arcsec}^{-2}\right)$ & $\begin{array}{l}\chi_{r}^{2} \\
(5)\end{array}$ & $\begin{array}{c}1 / n_{R} \\
(6)\end{array}$ & $\begin{array}{c}r_{R, 0} \\
(\operatorname{arcsec}) \\
(7)\end{array}$ & $\begin{array}{c}\mu_{R, 0} \\
\left(\mathrm{mag} \operatorname{arcsec}^{-2} \text { ) }\right. \\
(8)\end{array}$ & $\begin{array}{l}\chi_{r}^{2} \\
(9)\end{array}$ & $\begin{array}{c}1 / n_{\mathrm{H} \alpha} \\
(10)\end{array}$ & $\begin{array}{c}r_{\mathrm{H} \alpha, 0} \\
(\operatorname{arcsec}) \\
(11)\end{array}$ & $\begin{array}{c}\log f_{\mathrm{H} \alpha, 0} \\
\left(\operatorname{cgs} \operatorname{arcsec}^{-2}\right) \\
(12)\end{array}$ & $\begin{array}{c}\chi_{r}^{2} \\
(13)\end{array}$ & $\begin{array}{c}\text { Notes } \\
(14)\end{array}$ \\
\hline SBS $1428+457 \ldots \ldots \ldots$ & $0.05 \pm 0.19$ & $<0.01$ & $\ldots$ & 0.21 & $0.05 \pm 0.00$ & $<0.01$ & & 0.69 & $0.05 \pm 0.00$ & $<0.01$ & & 4.99 & \\
\hline Tol $1434+032 \ldots \ldots \ldots \ldots$ & $0.44 \pm 0.06$ & $0.27 \pm 0.24$ & $18.57 \pm 0.76$ & 0.61 & $0.87 \pm 0.25$ & $4.96 \pm 2.72$ & $21.59 \pm 1.90$ & 0.09 & $2.83 \pm 0.26$ & $20.98 \pm 0.33$ & $-16.17 \pm 0.04$ & 0.59 & b \\
\hline Mrk 475 & $0.71 \pm 0.15$ & $1.41 \pm 0.90$ & $20.36 \pm 2.19$ & 0.19 & $0.60 \pm 0.11$ & $0.91 \pm 0.64$ & $19.32 \pm 0.93$ & 0.16 & $0.05 \pm 0.00$ & $<0.01$ & & 3.10 & \\
\hline HS $1440+4302 \ldots \ldots \ldots$ & $0.73 \pm 0.41$ & $1.52 \pm 1.90$ & $22.10^{\mathrm{a}}$ & 0.40 & $0.52 \pm 0.18$ & $0.40 \pm 0.64$ & $19.24 \pm 4.44$ & 0.46 & $0.05 \pm 0.03$ & $<0.01$ & & 2.59 & \\
\hline HS $1442+4250 \ldots \ldots \ldots$ & $0.71 \pm 0.22$ & $1.58 \pm 1.67$ & $18.98 \pm 3.84$ & 0.06 & $0.64 \pm 0.18$ & $1.21 \pm 1.33$ & $18.21 \pm 2.62$ & 0.09 & $1.17 \pm 0.03$ & $11.45 \pm 0.21$ & $-15.05 \pm 0.02$ & 3.72 & \\
\hline UCM $1446+2312 \ldots .$. & $1.36 \pm 0.09$ & $7.93 \pm 0.62$ & $21.95 \pm 0.13$ & 1.28 & $1.32 \pm 0.06$ & $8.21 \pm 0.44$ & $20.94 \pm 0.12$ & 2.28 & & & $\ldots$ & & \\
\hline Tol $1448+116 \ldots \ldots \ldots . .$. & $1.11 \pm 0.81$ & $3.38 \pm 3.02$ & $21.82^{\mathrm{a}}$ & 0.20 & $0.08 \pm 0.19$ & $<0.01$ & & 0.31 & $0.05 \pm 0.19$ & $<0.01$ & & 0.16 & $\mathrm{~b}$ \\
\hline II Zw $70 \ldots \ldots \ldots \ldots \ldots$ & $0.20 \pm 0.03$ & $<0.01$ & $\ldots$ & 1.23 & $0.27 \pm 0.02$ & $<0.01$ & $12.45 \pm 0.93$ & 1.01 & $0.14 \pm 0.01$ & $<0.01$ & & 17.43 & \\
\hline II Zw 71 & $0.90 \pm 0.56$ & $5.22 \pm 6.24$ & $20.23^{\mathrm{a}}$ & 0.04 & $1.01 \pm 0.08$ & $8.02 \pm 1.15$ & $20.28 \pm 0.23$ & 0.09 & $1.82 \pm 0.03$ & $22.91 \pm 0.10$ & $-15.29 \pm 0.01$ & 32.37 & \\
\hline I Zw $115 .$. & $0.35 \pm 0.24$ & $0.02 \pm 1.17$ & $13.12^{\mathrm{a}}$ & 0.15 & $0.08 \pm 0.24$ & $<0.01$ & & 0.04 & & & & & \\
\hline SBS $1533+574 \ldots \ldots \ldots$ & $0.80 \pm 0.50$ & $1.59 \pm 2.17$ & $20.35^{\mathrm{a}}$ & 0.21 & $0.64 \pm 0.26$ & $0.85 \pm 1.21$ & $18.67 \pm 8.34$ & 0.23 & $0.05 \pm 0.08$ & $<0.01$ & & 0.98 & \\
\hline 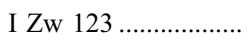 & $0.33 \pm 0.17$ & $0.01 \pm 0.32$ & $14.81^{\mathrm{a}}$ & 0.19 & $0.33 \pm 0.05$ & $0.01 \pm 0.03$ & $14.05 \pm 1.84$ & 0.15 & $0.05 \pm 0.00$ & $<0.01$ & & 1.02 & \\
\hline HS $1609+4827 \ldots \ldots \ldots$ & $0.69 \pm 0.09$ & $0.99 \pm 0.42$ & $18.33 \pm 0.58$ & 0.37 & $0.69 \pm 0.03$ & $1.05 \pm 0.17$ & $17.68 \pm 0.22$ & 1.48 & $1.05 \pm 0.03$ & $6.35 \pm 0.13$ & $-14.39 \pm 0.02$ & 28.17 & \\
\hline UCM $1612+1308 \ldots .$. & $0.05 \pm 0.04$ & $<0.01$ & $\ldots$ & 0.75 & $0.57 \pm 0.10$ & $0.22 \pm 0.16$ & $17.64 \pm 1.02$ & 0.03 & $1.07 \pm 0.02$ & $2.99 \pm 0.07$ & $-14.09 \pm 0.03$ & 6.99 & $\mathrm{~b}$ \\
\hline UGCA $412 \ldots \ldots \ldots \ldots . . . . .$. & $0.64 \pm 0.39$ & $1.57 \pm 3.07$ & $20.86^{\mathrm{a}}$ & 0.20 & $0.14 \pm 0.22$ & $<0.01$ & $\ldots$ & 0.11 & $0.54 \pm 1.30$ & $2.07 \pm 6.00$ & $-13.82^{\mathrm{a}}$ & 0.21 & \\
\hline HS $1704+4332 \ldots \ldots \ldots$ & $0.05 \pm 0.25$ & $<0.01$ & $\ldots$ & 0.21 & $0.05 \pm 0.44$ & $<0.01$ & & 0.05 & $0.45 \pm 0.03$ & $0.21 \pm 0.07$ & $-12.10 \pm 0.23$ & 4.69 & \\
\hline 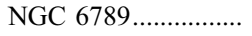 & $0.85 \pm 0.08$ & $8.93 \pm 1.92$ & $20.98 \pm 0.29$ & 0.08 & $0.77 \pm 0.09$ & $7.26 \pm 2.18$ & $19.69 \pm 0.39$ & 0.10 & $0.05 \pm 0.00$ & $<0.01$ & $\ldots$ & 5.39 & \\
\hline Tol 1924-416 ........... & $0.35 \pm 0.08$ & $0.03 \pm 0.09$ & $13.91 \pm 2.67$ & 0.22 & $0.30 \pm 0.06$ & $<0.01$ & $12.47 \pm 2.48$ & 0.30 & $0.05 \pm 0.00$ & $<0.01$ & & 7.14 & \\
\hline Tol 1937-423 ........... & $0.05 \pm 0.47$ & $<0.01$ & $\ldots$ & 0.11 & $0.06 \pm 0.40$ & $<0.01$ & & 0.24 & $0.05 \pm 1.02$ & $<0.01$ & & 0.08 & \\
\hline 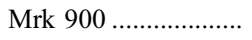 & $0.39 \pm 0.09$ & $0.17 \pm 0.37$ & $17.02 \pm 2.78$ & 0.07 & $0.33 \pm 0.15$ & $0.06 \pm 0.71$ & $15.85 \pm 9.70$ & 0.03 & $0.05 \pm 0.00$ & $<0.01$ & & 0.92 & \\
\hline Mrk 314 & $0.06 \pm 0.10$ & $<0.01$ & $\ldots$ & 0.19 & $0.05 \pm 0.22$ & $<0.01$ & & 0.02 & $2.13 \pm 0.72$ & $25.87 \pm 5.30$ & $-16.02 \pm 2.31$ & 0.17 & \\
\hline Mrk 324 & $0.41 \pm 0.21$ & $0.06 \pm 0.63$ & $14.89^{\mathrm{a}}$ & 0.54 & $0.90 \pm 0.30$ & $2.59 \pm 2.02$ & $19.48 \pm 5.95$ & 0.26 & $0.05 \pm 0.00$ & $<0.01$ & $\ldots$ & 1.08 & \\
\hline Mrk 328 & $0.41 \pm 0.18$ & $0.11 \pm 0.59$ & $16.61 \pm 8.60$ & 0.07 & $0.40 \pm 0.15$ & $0.10 \pm 0.45$ & $15.58 \pm 7.63$ & 0.07 & $0.05 \pm 0.00$ & $<0.01$ & & 3.46 & \\
\hline
\end{tabular}

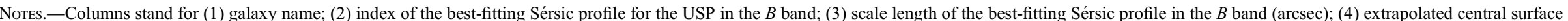

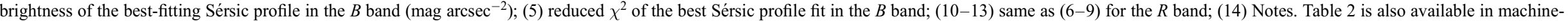
readable form in the electronic edition of the Astrophysical Journal Supplement.

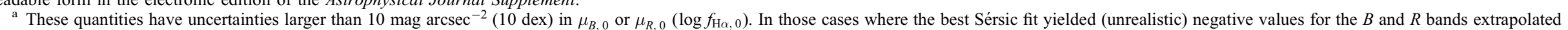
central surface brightness no data are given.

Errors in the points used for the fit are correlated. $\chi_{r}^{2}$ could be underestimated

Errors strongly correlated. The value of $\chi_{r}^{2}$ should be considered a lower limit (see text for details). 

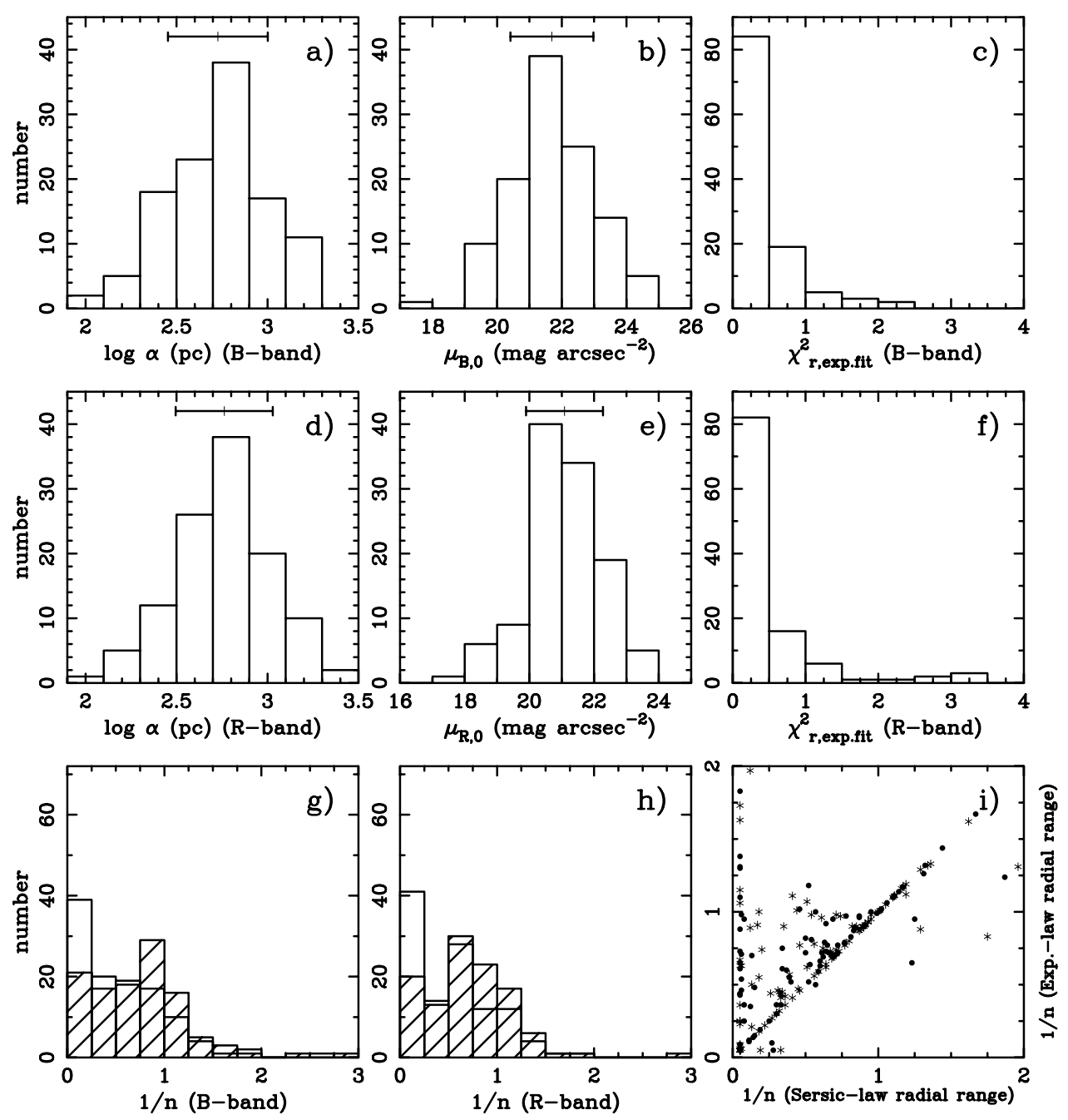

FIG. 2. - Histograms of the properties derived from the galaxies surface brightness profiles. (a) Scale length of the best-fitting exponential profile of the USP in the $B$ band. (b) Extrapolated central surface brightness of the best-fitting exponential profile in the $B$ band. ( $c$ ) Reduced $\chi^{2}$ of the best-fitting exponential profile in the $B$ band. $(d, e, f)$ The same as $(a, b, c)$ for the $R$ band. $(g)$ Best-fitting Sérsic index for the USP in the $B$ band. Hatched histograms represent the best-fitting Sérsic indices obtained using the same points in the profile that for the exponential-law fit. $(h)$ The same as $(g)$ for the $R$ band. $(i)$ Comparison between the Sérsic indices obtained using the radial ranges derived from the exponential-law and Sérsic-law fits for the $B$ (asterisks) and $R$ ( filled circles) bands. The tick marks shown in ( $a, b$, $d, e)$ represent the mean and mean $\pm 1 \sigma$ values of the corresponding distribution. [See the electronic edition of the Supplement for a color version of this figure.]

parameters on the chosen set of points considered during the fitting procedure (see $\S 3.2$; see also Cairós et al. 2003). Therefore, in the rest of the paper we will use the best-fitting exponential-law parameters derived in order to characterize the structural properties of the USP in BCDs. The use of very deep images at near-infrared wavelengths should improve significantly our knowledge about the detailed morphology of the USP in BCD galaxies given the smaller contamination associated with the HSB component at these wavelengths (see Noeske et al. 2003 for a pilot study using a sample of 12 BCDs).

\subsubsection{Structural Properties, Colors, and Luminosity}

The best-fit exponential and Sérsic-law parameters for the galaxies in the sample (including the $\chi_{r}^{2}$ of the best fit) are given in Tables 1 and 2, respectively. Table 3 shows the total, extrapolated $B$ and $R$ magnitudes of the best-fitting exponential USP. We also provide the color (observed and corrected for line and nebular-continuum contamination) of the USP weight averaged over the region where the best-fitting exponential and Sérsic laws were obtained.

The scale-length distributions obtained for the $B$ and $R$ bands are very similar, which confirms that the color gradients in the
USP, although present (see $\S 4.1$ ), are not very large. The mean values derived for the scale length of the USP are $\log (\alpha)=$ $2.7 \pm 0.3$ and $2.8 \pm 0.3$, respectively, for the $B$ - and $R$-band profiles. With regard to the extrapolated central surface brightness, the mean values obtained are $\mu_{B, 0}=21.7 \pm 1.3$ and $\mu_{R, 0}=21.1 \pm 1.2 \mathrm{mag} \operatorname{arcsec}^{-2}$. For comparison, Papaderos et al. (1996a) obtained a slightly brighter average value of $\mu_{B, 0}=21.3 \mathrm{mag} \operatorname{arcsec}^{-2}$ for their sample of $12 \mathrm{BCD}$ galaxies.

Figure 3 compares the integrated color of the galaxies in the sample (see Paper I) with the color of the USP (measured in the region where the best-fitting exponential law was derived). In most of the objects the $(B-R)$ color of the envelope is redder by about 0.3 mag than the integrated color, which has contributions from the recent star formation. Only in those objects where the line and nebular-continuum contamination is significant (e.g., UM 404, UM 417, HS 0822+3542, and I Zw 18) is the integrated color redder than the color of the USP (see Papaderos et al. 2002 for a detailed study of the impact of line and nebular-continuum contamination on the observed broadband properties of BCDs).

In Figure $4 a$ we compare the distribution of the galaxies in our sample in the $(B-R)-M_{B, \mathrm{LSB}}$ diagram. The $(B-R)$ 
TABLE 3

Properties of the Underlying Stellar Population

\begin{tabular}{|c|c|c|c|c|c|c|}
\hline $\begin{array}{c}\text { Object Name } \\
\text { (1) }\end{array}$ & $\begin{array}{c}B_{\mathrm{LSB}, \infty}^{\mathrm{E}} \\
(\mathrm{mag}) \\
(2)\end{array}$ & $\begin{array}{c}R_{\mathrm{LSB}, \infty}^{\mathrm{E}} \\
(\mathrm{mag}) \\
(3)\end{array}$ & $\begin{array}{c}(B-R)_{\mathrm{LSB}}^{\mathrm{E}} \\
(\mathrm{mag}) \\
(4)\end{array}$ & $\begin{array}{c}(B-R)_{\mathrm{LSB}}^{\mathrm{E}, \mathrm{c}} \\
(\mathrm{mag}) \\
(5)\end{array}$ & $\begin{array}{c}(B-R)_{\mathrm{LSB}}^{\mathrm{S}} \\
(\mathrm{mag}) \\
(6)\end{array}$ & $\begin{array}{c}(B-R)_{\mathrm{LSB}}^{\mathrm{S}, \mathrm{c}} \\
(\mathrm{mag}) \\
(7)\end{array}$ \\
\hline IC $10 \ldots \ldots$. & $5.47 \pm 0.10$ & $5.24 \pm 0.09$ & $0.23 \pm 0.14$ & $0.22 \pm 0.12$ & $0.23 \pm 0.14$ & $0.22 \pm 0.12$ \\
\hline HS $0029+1748 \ldots \ldots \ldots \ldots . . .$. & $17.38 \pm 0.24$ & $16.27 \pm 0.19$ & $0.66 \pm 0.16$ & $0.65 \pm 0.15$ & $0.56 \pm 0.15$ & $0.49 \pm 0.15$ \\
\hline Haro 14 & $13.44 \pm 0.11$ & $12.87 \pm 0.15$ & $0.90 \pm 0.15$ & $0.89 \pm 0.15$ & $0.85 \pm 0.15$ & $0.85 \pm 0.15$ \\
\hline UM $285 \ldots \ldots \ldots \ldots \ldots \ldots \ldots . .$. & $17.77 \pm 0.28$ & $18.04 \pm 0.36$ & $0.12 \pm 0.11$ & $0.14 \pm 0.08$ & $0.09 \pm 0.08$ & $0.14 \pm 0.08$ \\
\hline UCM $0049-0045 \ldots \ldots \ldots$. & $15.76 \pm 0.23$ & $14.42 \pm 0.28$ & $1.47 \pm 0.27$ & $1.40 \pm 0.25$ & $1.77 \pm 0.25$ & $1.78 \pm 0.25$ \\
\hline UM $323 \ldots \ldots \ldots \ldots \ldots \ldots \ldots$ & $15.81 \pm 0.19$ & $15.17 \pm 0.16$ & $0.73 \pm 0.09$ & $0.70 \pm 0.09$ & $0.73 \pm 0.09$ & $0.70 \pm 0.09$ \\
\hline Mrk 996 & $15.46 \pm 0.09$ & $14.51 \pm 0.16$ & $1.00 \pm 0.14$ & $1.00 \pm 0.14$ & $0.98 \pm 0.14$ & $0.98 \pm 0.14$ \\
\hline UM $133 \ldots \ldots \ldots \ldots \ldots \ldots . .$. & $16.06 \pm 0.16$ & $14.97 \pm 0.20$ & $1.17 \pm 0.15$ & $1.40 \pm 0.14$ & $1.16 \pm 0.15$ & $1.40 \pm 0.14$ \\
\hline 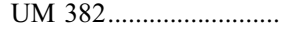 & $18.17 \pm 0.12$ & $18.08 \pm 0.30$ & $0.19 \pm 0.23$ & $0.14 \pm 0.22$ & $0.19 \pm 0.23$ & $0.14 \pm 0.22$ \\
\hline UM 404.............................. & $18.32 \pm 0.19$ & $18.85 \pm 0.37$ & $-0.52 \pm 0.14$ & $-0.80 \pm 0.13$ & $-0.52 \pm 0.14$ & $-0.80 \pm 0.13$ \\
\hline KUG 0207-016A......... & $15.50 \pm 0.14$ & $15.14 \pm 0.14$ & $0.51 \pm 0.13$ & $0.54 \pm 0.13$ & $0.51 \pm 0.13$ & $0.54 \pm 0.13$ \\
\hline 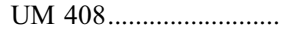 & $17.50 \pm 0.27$ & $16.68 \pm 0.14$ & $0.89 \pm 0.12$ & $0.87 \pm 0.12$ & $0.89 \pm 0.12$ & $0.87 \pm 0.12$ \\
\hline 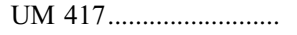 & $17.39 \pm 0.22$ & $17.69 \pm 0.15$ & $0.14 \pm 0.14$ & $0.05 \pm 0.14$ & $0.14 \pm 0.14$ & $0.05 \pm 0.14$ \\
\hline Mrk 370 & $13.93 \pm 0.05$ & $12.61 \pm 0.13$ & $1.31 \pm 0.13$ & $1.31 \pm 0.13$ & $1.30 \pm 0.13$ & $1.31 \pm 0.13$ \\
\hline 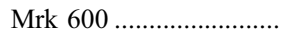 & $15.45 \pm 0.08$ & $15.17 \pm 0.17$ & $0.28 \pm 0.15$ & $0.26 \pm 0.15$ & $0.28 \pm 0.15$ & $0.26 \pm 0.15$ \\
\hline NGC $1522 \ldots \ldots \ldots \ldots \ldots \ldots . .$. & $14.82 \pm 0.03$ & $13.66 \pm 0.07$ & $1.13 \pm 0.06$ & $1.12 \pm 0.06$ & $1.09 \pm 0.06$ & $1.07 \pm 0.06$ \\
\hline NGC $1705 \ldots \ldots \ldots \ldots \ldots . .$. & $14.08 \pm 0.04$ & $12.89 \pm 0.07$ & $1.15 \pm 0.06$ & $1.17 \pm 0.06$ & $1.09 \pm 0.06$ & $1.11 \pm 0.06$ \\
\hline 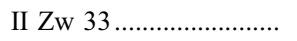 & $14.40 \pm 0.06$ & $13.77 \pm 0.18$ & $0.81 \pm 0.11$ & $0.79 \pm 0.11$ & $0.82 \pm 0.11$ & $0.81 \pm 0.11$ \\
\hline II Zw 33B.. & $16.91 \pm 0.28$ & $15.70 \pm 0.69$ & $1.35 \pm 0.28$ & $1.33 \pm 0.15$ & $1.13 \pm 0.14$ & $1.09 \pm 0.12$ \\
\hline II Zw 40 & $11.86 \pm 0.83$ & $11.02 \pm 0.14$ & $0.61 \pm 0.10$ & $0.54 \pm 0.09$ & $0.61 \pm 0.10$ & $0.54 \pm 0.09$ \\
\hline Tol $0610-387 \ldots \ldots \ldots \ldots . . .$. & $16.77 \pm 0.12$ & $15.63 \pm 0.07$ & $1.27 \pm 0.09$ & $1.28 \pm 0.08$ & $1.20 \pm 0.08$ & $1.20 \pm 0.08$ \\
\hline Mrk 5 & $15.44 \pm 0.13$ & $14.65 \pm 0.14$ & $0.88 \pm 0.13$ & $0.86 \pm 0.13$ & $0.88 \pm 0.13$ & $0.86 \pm 0.13$ \\
\hline Mrk 7 & $14.15 \pm 0.04$ & $13.50 \pm 0.19$ & $0.76 \pm 0.18$ & $0.73 \pm 0.18$ & $0.75 \pm 0.18$ & $0.73 \pm 0.18$ \\
\hline Mrk 86 & $12.79 \pm 0.11$ & $12.19 \pm 0.12$ & $1.01 \pm 0.14$ & $0.99 \pm 0.12$ & $0.96 \pm 0.13$ & $0.95 \pm 0.12$ \\
\hline HS $0822+3542 \ldots \ldots \ldots \ldots . .$. & $17.81 \pm 0.09$ & $18.19 \pm 0.18$ & $-0.38 \pm 0.14$ & $-0.51 \pm 0.14$ & $-0.36 \pm 0.14$ & $-0.50 \pm 0.14$ \\
\hline 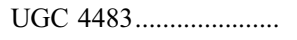 & $15.26 \pm 0.19$ & $14.30 \pm 0.23$ & $0.89 \pm 0.13$ & $1.00 \pm 0.13$ & $0.89 \pm 0.13$ & $1.00 \pm 0.13$ \\
\hline UGC 4703 N1 .................. & $17.15 \pm 0.16$ & $16.37 \pm 0.20$ & $0.76 \pm 0.06$ & $0.67 \pm 0.05$ & $0.80 \pm 0.06$ & $0.67 \pm 0.05$ \\
\hline UGC 4703 N2 .................. & $17.62 \pm 0.17$ & $16.69 \pm 0.21$ & $0.91 \pm 0.06$ & $0.88 \pm 0.05$ & $0.91 \pm 0.06$ & $0.88 \pm 0.05$ \\
\hline Mrk 1416 & $16.44 \pm 0.08$ & $15.90 \pm 0.13$ & $0.61 \pm 0.04$ & $0.53 \pm 0.04$ & $0.61 \pm 0.04$ & $0.53 \pm 0.04$ \\
\hline Mrk 108 & $15.73 \pm 0.13$ & $15.04 \pm 0.15$ & $0.68 \pm 0.09$ & $0.66 \pm 0.08$ & $0.59 \pm 0.08$ & $0.48 \pm 0.08$ \\
\hline Mrk 400 & $15.57 \pm 0.21$ & $14.91 \pm 0.19$ & $0.89 \pm 0.12$ & $1.07 \pm 0.12$ & $0.87 \pm 0.12$ & $1.07 \pm 0.12$ \\
\hline 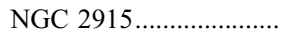 & $12.85 \pm 0.14$ & $11.53 \pm 0.47$ & $1.29 \pm 0.15$ & $1.30 \pm 0.09$ & $1.20 \pm 0.06$ & $1.21 \pm 0.06$ \\
\hline I Zw 18 & $17.49 \pm 0.19$ & $17.79 \pm 0.41$ & $-0.15 \pm 0.10$ & $-0.76 \pm 0.12$ & $0.08 \pm 0.14$ & $-0.53 \pm 0.14$ \\
\hline Mrk $1418 \ldots \ldots \ldots \ldots \ldots \ldots$ & $15.29 \pm 0.10$ & $13.66 \pm 0.21$ & $1.69 \pm 0.20$ & $1.78 \pm 0.20$ & $1.56 \pm 0.20$ & $1.58 \pm 0.20$ \\
\hline Mrk 1423 & $15.63 \pm 1.59$ & $14.24 \pm 0.19$ & $1.45 \pm 0.12$ & $1.42 \pm 0.11$ & $1.41 \pm 0.11$ & $1.41 \pm 0.11$ \\
\hline SBS $0940+544 C \ldots \ldots \ldots .$. & $16.92 \pm 0.09$ & $16.89 \pm 0.12$ & $0.14 \pm 0.11$ & $0.03 \pm 0.11$ & $0.14 \pm 0.11$ & $0.03 \pm 0.11$ \\
\hline Mrk 709 & $16.89 \pm 0.14$ & $16.02 \pm 0.12$ & $0.83 \pm 0.05$ & $0.82 \pm 0.04$ & $0.80 \pm 0.05$ & $0.77 \pm 0.04$ \\
\hline Mrk 1426 & $16.62 \pm 0.13$ & $16.55 \pm 0.19$ & $0.45 \pm 0.13$ & $0.46 \pm 0.12$ & $0.48 \pm 0.12$ & $0.48 \pm 0.12$ \\
\hline UGCA $184 \ldots \ldots \ldots \ldots \ldots . .$. & $16.85 \pm 0.15$ & $16.58 \pm 0.14$ & $0.28 \pm 0.12$ & $0.12 \pm 0.11$ & $0.21 \pm 0.11$ & $0.14 \pm 0.11$ \\
\hline 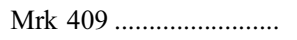 & $14.26 \pm 0.18$ & $13.31 \pm 0.14$ & $0.99 \pm 0.06$ & $0.99 \pm 0.06$ & $1.01 \pm 0.07$ & $1.00 \pm 0.06$ \\
\hline 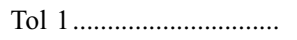 & $16.07 \pm 0.12$ & $15.79 \pm 0.13$ & $0.36 \pm 0.09$ & $0.30 \pm 0.09$ & $0.38 \pm 0.09$ & $0.30 \pm 0.09$ \\
\hline Tol $2 \ldots \ldots \ldots \ldots \ldots \ldots \ldots \ldots \ldots$ & $15.36 \pm 0.06$ & $14.35 \pm 0.07$ & $1.03 \pm 0.06$ & $1.03 \pm 0.06$ & $1.03 \pm 0.06$ & $1.03 \pm 0.06$ \\
\hline NGC $3125 \ldots \ldots \ldots \ldots \ldots \ldots \ldots$ & $15.00 \pm 0.15$ & $13.89 \pm 0.12$ & $1.15 \pm 0.10$ & $1.09 \pm 0.06$ & $1.06 \pm 0.06$ & $1.05 \pm 0.06$ \\
\hline SBS $1006+578 \ldots \ldots \ldots \ldots . .$. & $16.71 \pm 0.15$ & $15.97 \pm 0.16$ & $0.76 \pm 0.12$ & $0.75 \pm 0.12$ & $0.74 \pm 0.12$ & $0.75 \pm 0.12$ \\
\hline Haro 2 .............................. & $14.76 \pm 0.07$ & $13.91 \pm 0.12$ & $0.88 \pm 0.11$ & $0.87 \pm 0.11$ & $0.85 \pm 0.11$ & $0.85 \pm 0.11$ \\
\hline 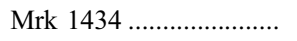 & $17.62 \pm 0.28$ & $17.22 \pm 0.29$ & $0.66 \pm 0.14$ & $0.54 \pm 0.14$ & $0.62 \pm 0.14$ & $0.48 \pm 0.14$ \\
\hline 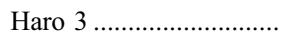 & $14.22 \pm 0.09$ & $13.15 \pm 0.15$ & $0.91 \pm 0.14$ & $0.90 \pm 0.14$ & $1.05 \pm 0.14$ & $1.06 \pm 0.14$ \\
\hline SBS $1054+504 \ldots \ldots \ldots \ldots . . .$. & $16.65 \pm 0.14$ & $15.93 \pm 0.18$ & $0.76 \pm 0.16$ & $0.74 \pm 0.15$ & $0.73 \pm 0.15$ & $0.73 \pm 0.15$ \\
\hline Haro 4 ............................. & $16.41 \pm 0.34$ & $15.97 \pm 0.24$ & $0.35 \pm 0.20$ & $0.23 \pm 0.19$ & $0.35 \pm 0.20$ & $0.23 \pm 0.19$ \\
\hline VII Zw $403 \ldots \ldots \ldots \ldots \ldots . . .$. & $14.69 \pm 0.26$ & $13.93 \pm 0.17$ & $0.80 \pm 0.18$ & $0.72 \pm 0.12$ & $0.79 \pm 0.12$ & $0.76 \pm 0.11$ \\
\hline Mrk 178 & $14.97 \pm 0.08$ & $14.11 \pm 0.10$ & $0.89 \pm 0.10$ & $0.88 \pm 0.10$ & $0.89 \pm 0.10$ & $0.88 \pm 0.10$ \\
\hline 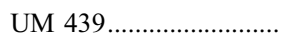 & $15.64 \pm 0.14$ & $14.78 \pm 0.12$ & $0.82 \pm 0.07$ & $0.81 \pm 0.07$ & $0.79 \pm 0.07$ & $0.78 \pm 0.07$ \\
\hline Mrk $1450 \ldots \ldots \ldots \ldots \ldots \ldots . .$. & $17.62 \pm 0.61$ & $16.57 \pm 0.51$ & $1.09 \pm 0.17$ & $0.99 \pm 0.11$ & $1.02 \pm 0.10$ & $1.02 \pm 0.10$ \\
\hline 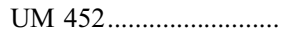 & $16.07 \pm 0.18$ & $14.76 \pm 0.15$ & $1.32 \pm 0.12$ & $1.32 \pm 0.12$ & $1.28 \pm 0.12$ & $1.28 \pm 0.12$ \\
\hline SBS $1147+520 \ldots \ldots \ldots \ldots$. & $18.76 \pm 0.43$ & $17.10 \pm 0.38$ & $1.34 \pm 0.19$ & $1.28 \pm 0.10$ & $1.34 \pm 0.19$ & $1.28 \pm 0.10$ \\
\hline Tol 17 ............................... & $16.62 \pm 0.13$ & $15.47 \pm 0.11$ & $1.18 \pm 0.08$ & $1.17 \pm 0.08$ & $1.15 \pm 0.08$ & $1.12 \pm 0.08$ \\
\hline 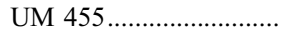 & $18.57 \pm 0.38$ & $17.34 \pm 0.17$ & $1.17 \pm 0.05$ & $1.07 \pm 0.03$ & $1.17 \pm 0.05$ & $1.07 \pm 0.03$ \\
\hline 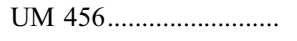 & $15.29 \pm 0.13$ & $14.83 \pm 0.09$ & $0.86 \pm 0.03$ & $0.84 \pm 0.03$ & $0.88 \pm 0.04$ & $0.84 \pm 0.03$ \\
\hline 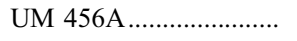 & $17.20 \pm 0.28$ & $17.24 \pm 0.34$ & $0.83 \pm 0.08$ & $0.75 \pm 0.04$ & $0.74 \pm 0.04$ & $0.69 \pm 0.03$ \\
\hline Pox 4 & $15.38 \pm 0.20$ & $15.20 \pm 0.16$ & $0.53 \pm 0.11$ & $0.52 \pm 0.11$ & $0.53 \pm 0.11$ & $0.52 \pm 0.11$ \\
\hline ESO $572-\mathrm{G} 025 \ldots \ldots \ldots \ldots$ & $16.17 \pm 0.06$ & $15.11 \pm 0.05$ & $1.09 \pm 0.05$ & $1.08 \pm 0.05$ & $1.13 \pm 0.05$ & $1.13 \pm 0.05$ \\
\hline VCC $0001 \ldots \ldots \ldots \ldots \ldots . .$. & $15.67 \pm 0.06$ & $14.51 \pm 0.05$ & $1.16 \pm 0.03$ & $1.16 \pm 0.03$ & $1.17 \pm 0.04$ & $1.16 \pm 0.03$ \\
\hline Mrk 1313 & $16.91 \pm 0.28$ & $16.34 \pm 0.17$ & $0.65 \pm 0.08$ & $0.62 \pm 0.06$ & $0.65 \pm 0.08$ & $0.62 \pm 0.06$ \\
\hline 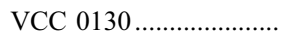 & $17.01 \pm 0.06$ & $16.19 \pm 0.03$ & $0.82 \pm 0.05$ & $0.77 \pm 0.05$ & $0.82 \pm 0.05$ & $0.77 \pm 0.05$ \\
\hline 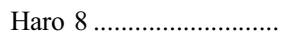 & $14.54 \pm 0.08$ & $13.45 \pm 0.14$ & $1.09 \pm 0.11$ & $1.09 \pm 0.11$ & $1.09 \pm 0.11$ & $1.09 \pm 0.11$ \\
\hline UM 491 ................................. & $16.59 \pm 0.30$ & $15.76 \pm 0.19$ & $0.91 \pm 0.09$ & $0.89 \pm 0.08$ & $0.91 \pm 0.09$ & $0.89 \pm 0.08$ \\
\hline
\end{tabular}


TABLE 3-Continued

\begin{tabular}{|c|c|c|c|c|c|c|}
\hline $\begin{array}{c}\text { Object Name } \\
\text { (1) }\end{array}$ & $\begin{array}{c}B_{\mathrm{LSB}, \infty}^{\mathrm{E}} \\
(\mathrm{mag}) \\
(2)\end{array}$ & $\begin{array}{c}R_{\mathrm{LSB}, \infty}^{\mathrm{E}} \\
(\mathrm{mag}) \\
(3)\end{array}$ & $\begin{array}{c}(B-R)_{\mathrm{LSB}}^{\mathrm{E}} \\
(\mathrm{mag}) \\
(4)\end{array}$ & $\begin{array}{c}(B-R)_{\mathrm{LSB}}^{\mathrm{E}, \mathrm{c}} \\
(\mathrm{mag}) \\
(5)\end{array}$ & $\begin{array}{c}(B-R)_{\mathrm{LSB}}^{\mathrm{S}} \\
(\mathrm{mag}) \\
(6)\end{array}$ & $\begin{array}{c}(B-R)_{\mathrm{LSB}}^{\mathrm{S}, \mathrm{c}} \\
(\mathrm{mag}) \\
(7)\end{array}$ \\
\hline ISZ $399 \ldots \ldots \ldots$. & $15.12 \pm 0.02$ & $13.80 \pm 0.06$ & $1.32 \pm 0.06$ & $1.32 \pm 0.06$ & $1.32 \pm 0.06$ & $1.32 \pm 0.06$ \\
\hline VCC $0459 .$. & $15.63 \pm 0.11$ & $14.61 \pm 0.11$ & $1.06 \pm 0.05$ & $1.05 \pm 0.05$ & $1.06 \pm 0.05$ & $1.05 \pm 0.05$ \\
\hline VCC 0655 & $13.71 \pm 0.07$ & $12.41 \pm 0.05$ & $1.29 \pm 0.05$ & $1.30 \pm 0.05$ & $1.30 \pm 0.05$ & $1.30 \pm 0.05$ \\
\hline Tol 65 & $17.79 \pm 0.27$ & $17.38 \pm 0.22$ & $0.44 \pm 0.05$ & $0.50 \pm 0.05$ & $0.44 \pm 0.05$ & $0.50 \pm 0.05$ \\
\hline VCC 0848 & $15.46 \pm 0.08$ & $14.48 \pm 0.05$ & $0.95 \pm 0.05$ & $0.94 \pm 0.05$ & $0.95 \pm 0.05$ & $0.94 \pm 0.05$ \\
\hline Mrk 209. & $15.13 \pm 0.12$ & $14.47 \pm 0.33$ & $0.59 \pm 0.15$ & $0.54 \pm 0.13$ & $0.56 \pm 0.13$ & $0.53 \pm 0.13$ \\
\hline Mrk 1329. & $14.52 \pm 0.08$ & $13.60 \pm 0.10$ & $0.90 \pm 0.09$ & $0.90 \pm 0.09$ & $0.89 \pm 0.09$ & $0.88 \pm 0.09$ \\
\hline UGCA $290 \ldots \ldots \ldots \ldots . . . .$. & $15.36 \pm 0.06$ & $14.88 \pm 0.15$ & $0.60 \pm 0.14$ & $0.57 \pm 0.13$ & $0.62 \pm 0.14$ & $0.59 \pm 0.13$ \\
\hline VCC $1750 \ldots \ldots$ & $17.51 \pm 0.12$ & $16.45 \pm 0.08$ & $1.08 \pm 0.06$ & $1.07 \pm 0.06$ & $1.08 \pm 0.06$ & $1.08 \pm 0.06$ \\
\hline 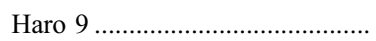 & $13.64 \pm 0.05$ & $12.72 \pm 0.10$ & $0.95 \pm 0.11$ & $0.98 \pm 0.11$ & $0.95 \pm 0.11$ & $0.98 \pm 0.11$ \\
\hline 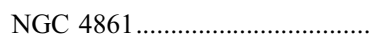 & $12.89 \pm 0.04$ & $12.03 \pm 0.09$ & $0.84 \pm 0.09$ & $0.76 \pm 0.09$ & $0.84 \pm 0.09$ & $0.76 \pm 0.09$ \\
\hline 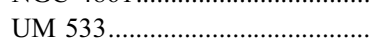 & $15.06 \pm 0.10$ & $13.94 \pm 0.12$ & $1.10 \pm 0.11$ & $1.06 \pm 0.11$ & $1.09 \pm 0.11$ & $1.06 \pm 0.11$ \\
\hline Mrk $450 \ldots .$. & $15.21 \pm 0.10$ & $14.31 \pm 0.12$ & $0.96 \pm 0.12$ & $0.95 \pm 0.11$ & $0.87 \pm 0.11$ & $0.86 \pm 0.11$ \\
\hline NGC 5058 & $14.59 \pm 0.12$ & $13.87 \pm 0.10$ & $0.79 \pm 0.08$ & $0.79 \pm 0.08$ & $0.78 \pm 0.08$ & $0.77 \pm 0.08$ \\
\hline PGC 046448 & $16.58 \pm 0.13$ & $15.54 \pm 0.18$ & $1.18 \pm 0.04$ & $1.16 \pm 0.03$ & $1.18 \pm 0.03$ & $1.18 \pm 0.03$ \\
\hline Pox $186 \ldots \ldots$ & $19.14 \pm 0.20$ & $18.67 \pm 0.46$ & $0.79 \pm 0.14$ & $0.75 \pm 0.09$ & $0.60 \pm 0.05$ & $0.49 \pm 0.03$ \\
\hline Tol $35 \ldots \ldots \ldots . . .$. & $14.19 \pm 0.09$ & $13.25 \pm 0.07$ & $1.03 \pm 0.06$ & $1.04 \pm 0.06$ & $1.03 \pm 0.06$ & $1.03 \pm 0.06$ \\
\hline SBS $1331+493 \ldots \ldots \ldots$ & $14.78 \pm 0.09$ & $14.22 \pm 0.19$ & $0.95 \pm 0.18$ & $0.91 \pm 0.17$ & $0.77 \pm 0.17$ & $0.78 \pm 0.17$ \\
\hline Tol $85 \ldots \ldots \ldots$ & $16.03 \pm 0.21$ & $16.03 \pm 0.22$ & $0.56 \pm 0.04$ & $0.49 \pm 0.03$ & $0.58 \pm 0.05$ & $0.50 \pm 0.03$ \\
\hline 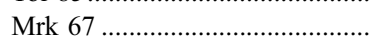 & $17.44 \pm 0.19$ & $16.21 \pm 0.22$ & $1.28 \pm 0.10$ & $1.21 \pm 0.09$ & $1.20 \pm 0.09$ & $1.19 \pm 0.09$ \\
\hline Mrk 1480 & $17.56 \pm 0.25$ & $16.52 \pm 0.23$ & $0.99 \pm 0.09$ & $0.94 \pm 0.08$ & $0.95 \pm 0.08$ & $0.94 \pm 0.08$ \\
\hline Mrk 1481 & $17.00 \pm 0.14$ & $16.00 \pm 0.10$ & $1.00 \pm 0.08$ & $1.17 \pm 0.07$ & $0.97 \pm 0.08$ & $1.14 \pm 0.07$ \\
\hline Tol $1345-420 \ldots \ldots$ & $16.77 \pm 0.36$ & $16.29 \pm 0.24$ & $0.59 \pm 0.10$ & $0.50 \pm 0.06$ & $1.02 \pm 0.05$ & $0.95 \pm 0.05$ \\
\hline HS $1400+3927 \ldots \ldots \ldots \ldots \ldots$ & $17.77 \pm 0.10$ & $16.48 \pm 0.14$ & $1.26 \pm 0.12$ & $1.17 \pm 0.12$ & $1.23 \pm 0.12$ & $1.18 \pm 0.12$ \\
\hline SBS $1415+437 \ldots \ldots \ldots$ & $15.68 \pm 0.09$ & $14.99 \pm 0.15$ & $0.70 \pm 0.13$ & $0.66 \pm 0.13$ & $0.72 \pm 0.13$ & $0.69 \pm 0.13$ \\
\hline SBS $1428+457 \ldots \ldots$ & $16.34 \pm 0.28$ & $15.45 \pm 0.34$ & $0.98 \pm 0.11$ & $0.98 \pm 0.10$ & $0.98 \pm 0.11$ & $0.98 \pm 0.10$ \\
\hline Tol $1434+032 \ldots \ldots \ldots$ & $16.27 \pm 0.11$ & $15.87 \pm 0.09$ & $0.42 \pm 0.08$ & $0.53 \pm 0.07$ & $0.41 \pm 0.08$ & $0.51 \pm 0.07$ \\
\hline Mrk $475 \ldots \ldots \ldots \ldots$ & $16.92 \pm 0.10$ & $16.24 \pm 0.12$ & $0.91 \pm 0.08$ & $0.88 \pm 0.08$ & $0.81 \pm 0.08$ & $0.79 \pm 0.08$ \\
\hline HS $1440+4302$. & $18.50 \pm 0.20$ & $17.15 \pm 0.17$ & $1.38 \pm 0.13$ & $1.32 \pm 0.12$ & $1.37 \pm 0.12$ & $1.34 \pm 0.12$ \\
\hline HS $1442+4250 \ldots \ldots$ & $15.42 \pm 0.07$ & $14.84 \pm 0.08$ & $0.66 \pm 0.09$ & $0.64 \pm 0.09$ & $0.69 \pm 0.09$ & $0.67 \pm 0.09$ \\
\hline UCM $1446+2312 \ldots \ldots \ldots \ldots \ldots \ldots$ & $15.77 \pm 0.14$ & $14.70 \pm 0.16$ & $1.10 \pm 0.09$ & $1.19 \pm 0.09$ & $1.10 \pm 0.09$ & $1.19 \pm 0.09$ \\
\hline 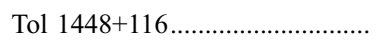 & $17.30 \pm 0.38$ & $16.52 \pm 0.20$ & $0.87 \pm 0.13$ & $0.86 \pm 0.12$ & $0.89 \pm 0.14$ & $0.88 \pm 0.13$ \\
\hline II Zw 70 & $16.57 \pm 0.27$ & $15.53 \pm 0.19$ & $0.99 \pm 0.18$ & $0.94 \pm 0.18$ & $0.89 \pm 0.18$ & $0.93 \pm 0.18$ \\
\hline 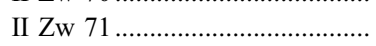 & $14.53 \pm 0.16$ & $13.78 \pm 0.12$ & $0.94 \pm 0.19$ & $0.94 \pm 0.19$ & $0.94 \pm 0.19$ & $0.94 \pm 0.19$ \\
\hline I Zw 115 & $15.02 \pm 0.11$ & $14.08 \pm 0.09$ & $1.04 \pm 0.12$ & $1.06 \pm 0.12$ & $1.13 \pm 0.12$ & $1.13 \pm 0.12$ \\
\hline SBS $1533+574 \ldots \ldots$ & $16.98 \pm 0.23$ & $16.02 \pm 0.14$ & $1.09 \pm 0.15$ & $1.10 \pm 0.14$ & $1.09 \pm 0.14$ & $1.10 \pm 0.14$ \\
\hline I Zw 123 & $16.75 \pm 0.22$ & $15.85 \pm 0.15$ & $1.11 \pm 0.14$ & $1.11 \pm 0.13$ & $1.07 \pm 0.13$ & $1.07 \pm 0.13$ \\
\hline HS $1609+4827 \ldots \ldots \ldots$ & $15.50 \pm 0.16$ & $14.73 \pm 0.13$ & $0.80 \pm 0.14$ & $0.79 \pm 0.14$ & $0.80 \pm 0.14$ & $0.79 \pm 0.14$ \\
\hline 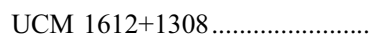 & $17.99 \pm 0.80$ & $17.58 \pm 0.10$ & $0.68 \pm 0.23$ & $0.56 \pm 0.24$ & $0.79 \pm 0.16$ & $0.72 \pm 0.16$ \\
\hline UGCA $412 \ldots \ldots \ldots$ & $16.80 \pm 0.16$ & $15.55 \pm 0.21$ & $1.24 \pm 0.11$ & $1.09 \pm 0.10$ & $1.24 \pm 0.11$ & $1.09 \pm 0.10$ \\
\hline 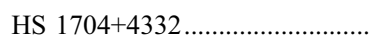 & $18.80 \pm 0.42$ & $18.82 \pm 0.28$ & $0.40 \pm 0.20$ & $0.57 \pm 0.09$ & $0.41 \pm 0.11$ & $0.40 \pm 0.09$ \\
\hline NGC $6789 \ldots \ldots \ldots$ & $13.94 \pm 0.05$ & $12.85 \pm 0.09$ & $1.09 \pm 0.08$ & $1.13 \pm 0.08$ & $1.09 \pm 0.08$ & $1.12 \pm 0.08$ \\
\hline 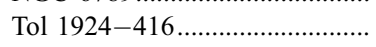 & $16.20 \pm 0.29$ & $14.68 \pm 0.14$ & $0.91 \pm 0.06$ & $0.89 \pm 0.05$ & $0.79 \pm 0.05$ & $0.75 \pm 0.05$ \\
\hline 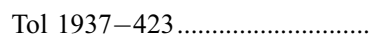 & $16.97 \pm 0.72$ & $15.39 \pm 0.25$ & $1.21 \pm 0.11$ & $1.21 \pm 0.06$ & $1.19 \pm 0.07$ & $1.19 \pm 0.05$ \\
\hline Mrk 900 & $14.91 \pm 0.08$ & $13.97 \pm 0.16$ & $0.77 \pm 0.13$ & $1.08 \pm 0.13$ & $0.81 \pm 0.13$ & $1.08 \pm 0.13$ \\
\hline Mrk 314 & $14.73 \pm 0.12$ & $14.06 \pm 0.30$ & $0.64 \pm 0.27$ & $0.64 \pm 0.26$ & $0.63 \pm 0.24$ & $0.62 \pm 0.24$ \\
\hline Mrk 324 & $16.12 \pm 0.23$ & $15.31 \pm 0.23$ & $0.71 \pm 0.14$ & $0.71 \pm 0.14$ & $0.71 \pm 0.14$ & $0.71 \pm 0.14$ \\
\hline Mrk 328 & $15.83 \pm 0.14$ & $14.87 \pm 0.13$ & $0.93 \pm 0.11$ & $0.92 \pm 0.10$ & $0.92 \pm 0.10$ & $0.92 \pm 0.10$ \\
\hline
\end{tabular}

Notes.-Columns stand for (1) galaxy name; (2) total $B$ band magnitude of the best-fitting exponential profile fitted to the USP surface brightness distribution (corrected for Galactic extinction); (3) same as (2) for the $R$ band; (4) $(B-R$ ) color of the underling stellar population in the region where the best-fitting exponential profile was derived; (5) same as (4) corrected for line and nebular-continuum contamination; $(6)(B-R)$ color of the underling stellar population in the region where the best-fitting Sérsic profile was derived; (7) same as (6) corrected for line and nebular-continuum contamination. Table 3 is also available in machine-readable form in the electronic edition of the Astrophysical Journal Supplement.

colors in this figure have been corrected for line and nebularcontinuum contamination, and the absolute magnitude plotted $\left(M_{B, \mathrm{LSB}}\right)$ refers to that of the best-fitting exponential LSB component associated with the USP. This plot constitutes a direct means of comparing the properties of the USP of BCD galaxies with those of other types of dwarf galaxies.

Since local dwarf elliptical galaxies (dE) show no significant star formation and very shallow color gradients (e.g., Vader et al.
1988) we can directly compare this plot with the (integrated) color-magnitude diagram of dE galaxies (Fig. $4 a$, circles). Field dE galaxies (Parodi et al. 2002), dE in the Sculptor and Cen A groups (Jerjen et al. 2000), and cluster dE in Virgo (Barazza et al. 2003) and Perseus (Conselice et al. 2003) are plotted. We also show the fiducial color-magnitude relationship for the Coma cluster (solid line; Secker et al. 1997). Finally, we have also included in this plot a small sample of dwarf irregular galaxies 


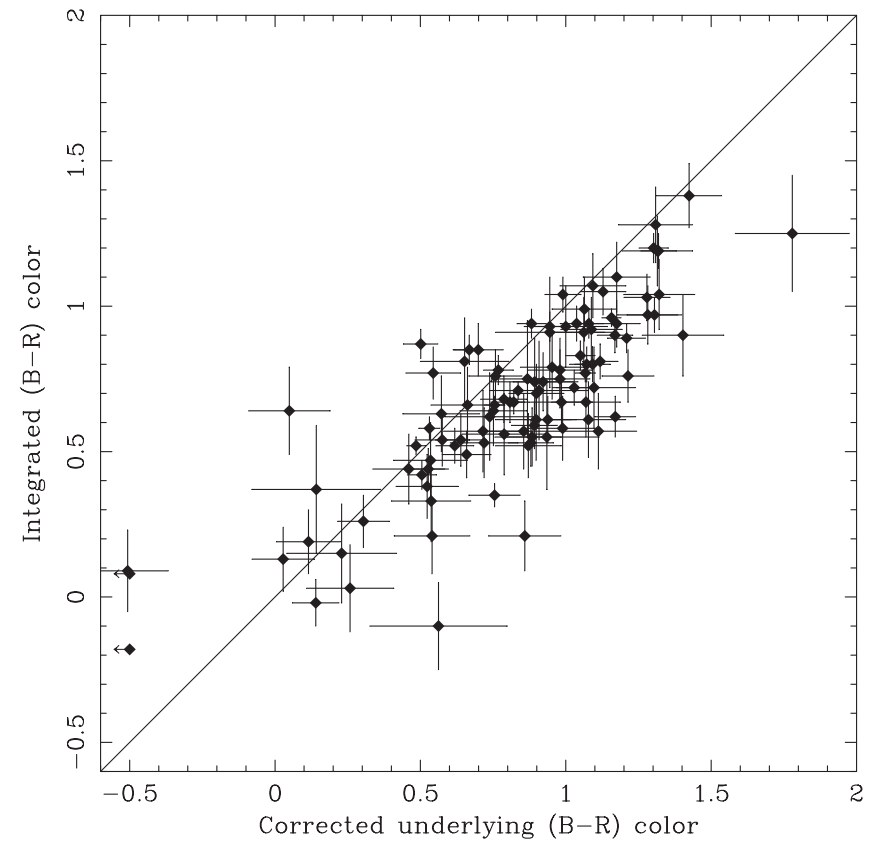

FIG. 3.-Comparison between the integrated $(B-R)$ color (from Paper I) of the galaxies and the color of their USP.

for which colors and luminosities of their USP are available (open stars; Parodi et al. 2002).

Figure $4 a$ shows that there are many BCD galaxies in our sample that show colors and luminosities of their USP that are comparable to those of elliptical galaxies. In Figure $4 b$ the frequency histograms of the $(B-R)$ color of dE galaxies (open histogram) and BCD galaxies (solid and hatched histograms) are plotted. While there is significant overlap, the USP of BCDs is (on average) bluer than that of $\mathrm{dE}$ galaxies. However, Figure $4 b$ also shows that the distribution of the color of the USP of BCD galaxies with smooth envelopes ("E" type; $\mathrm{nE}$ and $\mathrm{iE}$ types according to Loose \& Thuan 1985) (solid histogram) is more similar to that of $\mathrm{dE}$ galaxies than the one for $\mathrm{BCD}$ galaxies with irregular envelopes (iI-type BCDs; hatched histogram).

These results suggest that a measurable fraction of the $\mathrm{BCD}$ galaxies show USP with colors, luminosities, and apparently also morphologies similar to those of dwarf ellipticals.

In order to quantitatively analyze the morphological differences between BCD galaxies with red and blue USP we also compare the extrapolated central surface brightness and scale lengths of the galaxies in our sample (see Table 1) with those of $\mathrm{BCD}$, dI, and dE galaxies taken from the literature (P. Papaderos 2004, private communication; see also Papaderos et al. 1996a). In Figure $5 a$ we plot the extrapolated central surface brightness against the luminosity (both in the $B$ band) of the best-fitting exponential USP for the galaxies in our sample and compare them with those of dE (small dots), dI (stars), LSB (open crosses), and other BCD galaxies from the literature (open diamonds). Those BCDs in our sample offset by less than $\pm 0.4 \mathrm{mag}$ from the color-magnitude relationship of $\mathrm{dE}$ galaxies are represented by large dots. BCDs offset by more than this amount are shown as filled diamonds. This allows us to separate BCD galaxies with red envelopes from those with blue envelopes. The horizontal dotted line at $\mu_{B, 0}=22 \mathrm{mag} \operatorname{arcsec}^{-2}$ marks the separation between BCDs and other types of dwarf galaxies, as proposed by Papaderos et al. (1996a). This figure shows that there are many $\mathrm{BCD}$ galaxies with a central surface brightness of

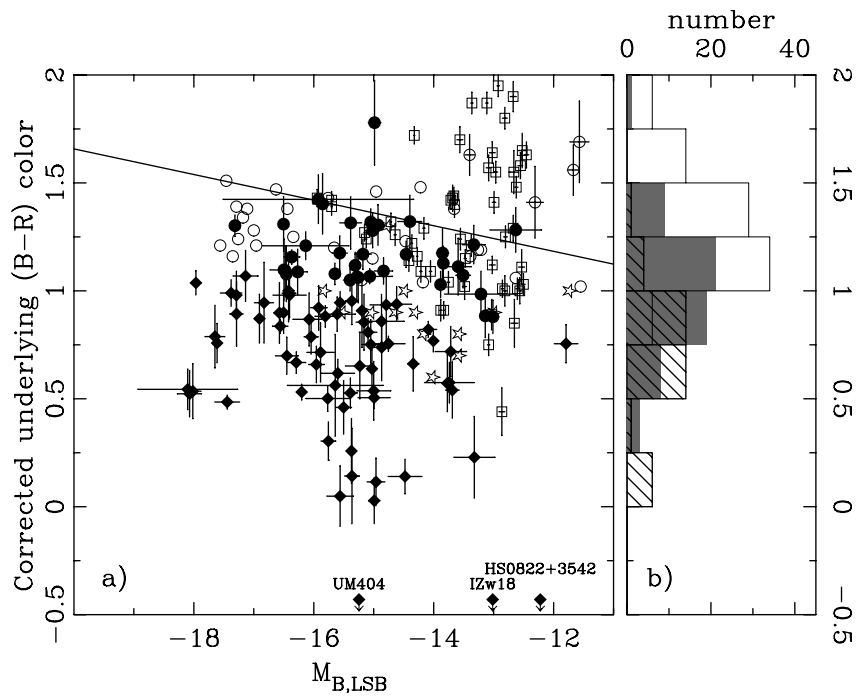

FIG. 4.- (a) Comparison between the $(B-R)$ color of the USP and its absolute magnitude computed from the best exponential fit to the surfacebrightness profile of the USP. BCD galaxies in our sample having blue (red) envelopes are represented by filled diamonds (dots) (see text for more details). Dwarf elliptical (open circles and squares) and dwarf irregular galaxies (stars) from the literature are also plotted. The solid line represents the color-magnitude relationship of dwarf elliptical galaxies in the Coma cluster (Secker et al. 1997). Note that the galaxies from Conselice et al. (2003) (open squares) were classified as dwarf ellipticals based exclusively on their structural properties. (b) Histogram of the $(B-R)$ color of the USP for "E"-type ( filled histogram) and "I"-type (cross-hatched histogram) BCDs in our sample and the reference sample of dwarf ellipticals (open histogram). [See the electronic edition of the Supplement for a color version of this figure.]

the USP fainter than $22 \mathrm{mag} \operatorname{arcsec}^{-2}$ and that in most of those galaxies this component is as red as $\mathrm{dE}$ galaxies.

Figure $5 b$ shows the exponential scale length plotted against luminosity for the same objects as in Figure $5 a$. Again, BCD galaxies with red envelopes show (on average) scale lengths comparable to those seen in $\mathrm{dE}$ galaxies, and larger than those of BCDs with blue envelopes. The dashed line shows the least-squares fit to the distribution of dwarf elliptical galaxies in this plot.

By analyzing the properties of the USP of the galaxies individually we find that a total of 17 BCDs in our sample show envelopes with (1) smooth elliptical morphologies (nE or iE types), (2) dE-like colors, and (3) faint extrapolated central surface brightnesses. This makes up slightly over $15 \%$ of the whole sample.

\subsection{Implications on the Unified Evolutionary Model of Dwarf Galaxies}

The fraction of BCDs with properties of their USP similar to those of dwarf ellipticals may be even larger if, as proposed by Papaderos et al. (1996a), the structural properties of the USP of BCDs may vary with time in response to changes in the gravitational potential driven by the collective effect of stellar winds from massive stars and supernova explosions (see next section for a discussion on this topic).

The results presented above allow us to conclude that a significant fraction of the BCDs in the nearby universe (at least $15 \%$ ) are consistent with being dwarf elliptical galaxies that are now experiencing, or have recently experienced, an episode of active star formation. These objects can be easily identified with the "slowly moving," gas-accreting $\mathrm{dE}$ galaxies proposed by Silk et al. (1987). According to this scenario, these galaxies 

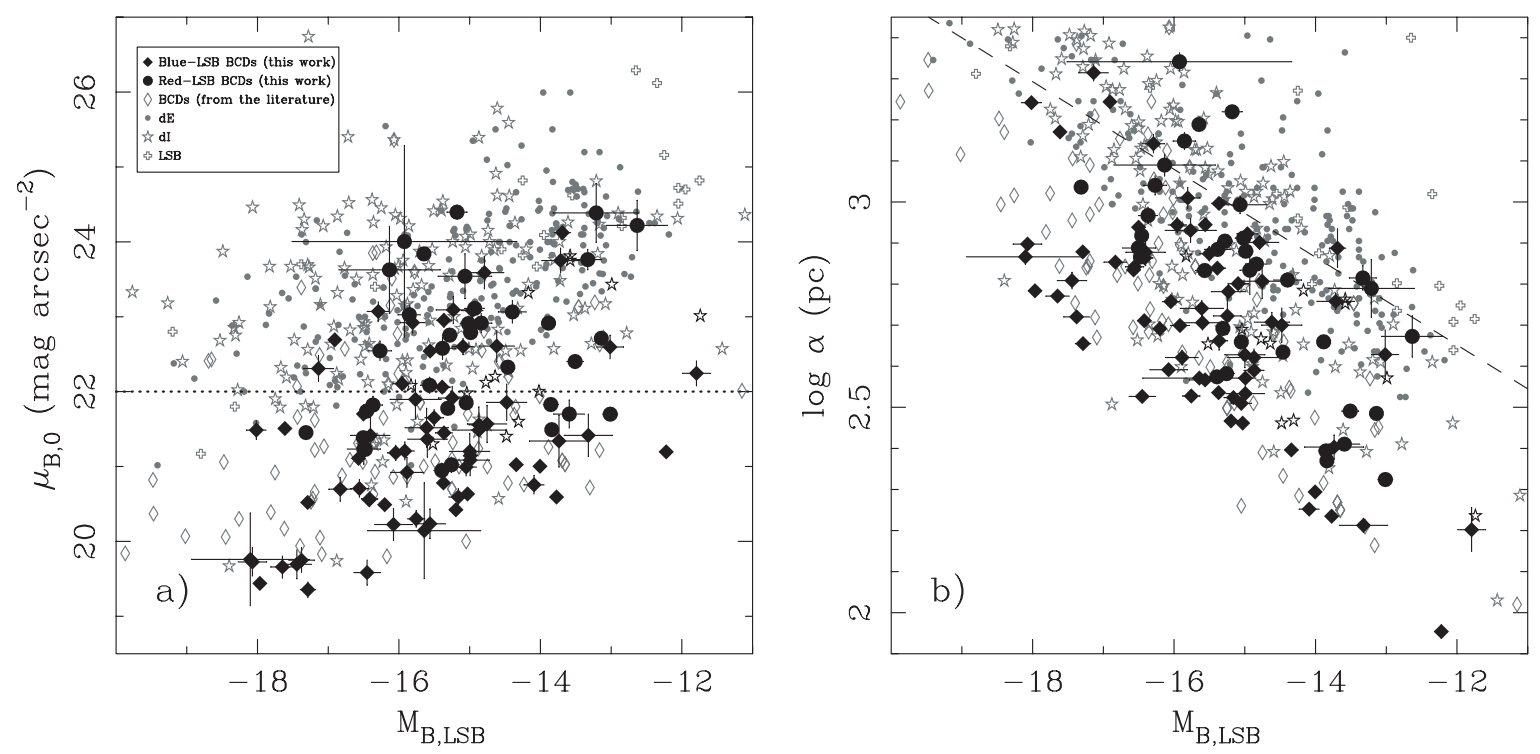

FIG. 5.-Correlations between the structural properties of the USP and luminosity. BCD galaxies in our sample having blue (red) envelopes are represented by filled diamonds (dots) (see text for more details). The properties of dE (small dots), dI (stars), LSB (open crosses), and other BCD galaxies from the literature (open diamonds) are also shown. [See the electronic edition of the Supplement for a color version of this figure.]

are expected to subsequently evolve into nucleated dwarf ellipticals, following the sequence $\mathrm{dE} \rightarrow \mathrm{BCD} \rightarrow \mathrm{dE}, \mathrm{N}$. Note that the possible evolution from BCDs to (nucleated) $\mathrm{dE}$ galaxies has been recently questioned by Tajiri \& Kamaya (2002; see also van Zee et al. 2001) because of the difficulty in having these galaxies blow away their $\mathrm{H}$ i envelopes.

\subsection{Impact of the Recent Star Formation on the Evolution of BCDs}

In this section we study the effect of the current star formation on the structural properties of the USP of BCDs. According to Papaderos et al. (1996a) the offset between the scale length of $\mathrm{dE}$ and the USP of some BCDs may be due to expansion of the USP in response to changes in the gravitational field produced by collective supernova-driven winds. Here we define the "degree of expansion" as the difference (in logarithmic scale) between the scale length of the galaxy and the average scale length of dwarf ellipticals of the same luminosity. The latter quantity is obtained from the least-squares fit to the dE's scale length and luminosity shown by the dashed line in Figure $5 b$. In Figure 6 the degree of expansion is compared with the observed equivalent width of $\mathrm{H} \alpha$ (Fig. $6 a$ ) and with the difference between the galaxy color with that of its USP (Fig. 6b). Note that the errors in the degree of expansion include the scatter in the least-squares fit used to derive the average scale length of dwarf ellipticals at a given luminosity.

Despite the strong dependence of the equivalent width of $\mathrm{H} \alpha$ on the age of the young stellar population for the case of instantaneous star formation, if the expansion of the USP in BCDs is related to the strength of the recent star-forming event we would expect to find a correlation between $\operatorname{EW}(\mathrm{H} \alpha)$ and the degree of expansion (as defined above). This correlation should be even more evident if, as it is thought, the recent star formation in BCDs takes place in episodes of approximately constant star formation that last as long as $10^{8} \mathrm{yr}$ (see Papaderos et al. 2002 and references therein). However, Figure $6 a$ does not show any obvious correlation between $\mathrm{EW}(\mathrm{H} \alpha)$ and the degree of expansion, which suggests that the current episode of star formation probably has had little impact, if any, on the structural properties of the USP.

The $(B-R)$ color is even more sensitive to the strength of the most recent star formation episode than is the equivalent width of $\mathrm{H} \alpha$ (see Fig. $6 a$ of Paper I). Thus, for a relatively evolved USP, a recent episode of star formation with even a small burst strength may have a strong impact on the observed $(B-R)$ color of the galaxy for a relatively long period of time. However, in the case of BCD galaxies with blue envelopes the difference in color between the galaxy and its USP may be small even for relatively massive bursts.

Figure $6 b$ seems to shows a slight tendency for galaxies with more negative degree of expansion to have slightly smaller differences in color between the galaxy and its USP. This is opposite to what we would expect if both the degree of expansion and the difference in color measured would only depend on the burst strength of the most recent star formation event.

In order to quantify the impact of this recent star formation on the structural properties of the USP of BCDs we will use the formalism of Papaderos et al. (1996a). According to these authors we can write the degree of expansion as

$$
\log (\alpha)-\left\langle\log \left(\alpha_{\mathrm{dE}}\right)\right\rangle=\log \left(1-\frac{F_{0}}{1+\psi_{R_{\mathrm{H}}}}\right),
$$

where $F_{0}$ is the fraction of the visible mass ejected from the galaxy as a consequence of the collective effect of supernovadriven winds, and $\psi_{R_{\mathrm{H}}}$ is the dark-to-visible mass ratio inside the galaxy's Holmberg radius $\left(R_{\mathrm{H} 0}\right)$. Note that in our case $F_{0}$ is defined to be positive. The simulations of the evolution of the ISM around dwarf starburst galaxies carried out by Mac Low \& Ferrara (1999) indicate that $F_{0}$ is a strong function of the total visible mass of the galaxy $\left(M_{\mathrm{vis}}\right)$ and the kinetic energy injection rate $\left(L_{\mathrm{kin}}\right)$. We have used the $F_{0}$ values given by these authors for visible masses between $10^{6}$ and $10^{9} M_{\odot}$ and kinetic luminosities in the range $10^{37}-10^{39} \mathrm{ergs} \mathrm{s}^{-1}$. The fraction of mass ejected in the case of kinetic luminosities greater than $10^{39} \mathrm{ergs}$ $\mathrm{s}^{-1}$ has been determined using the following relationship, which 


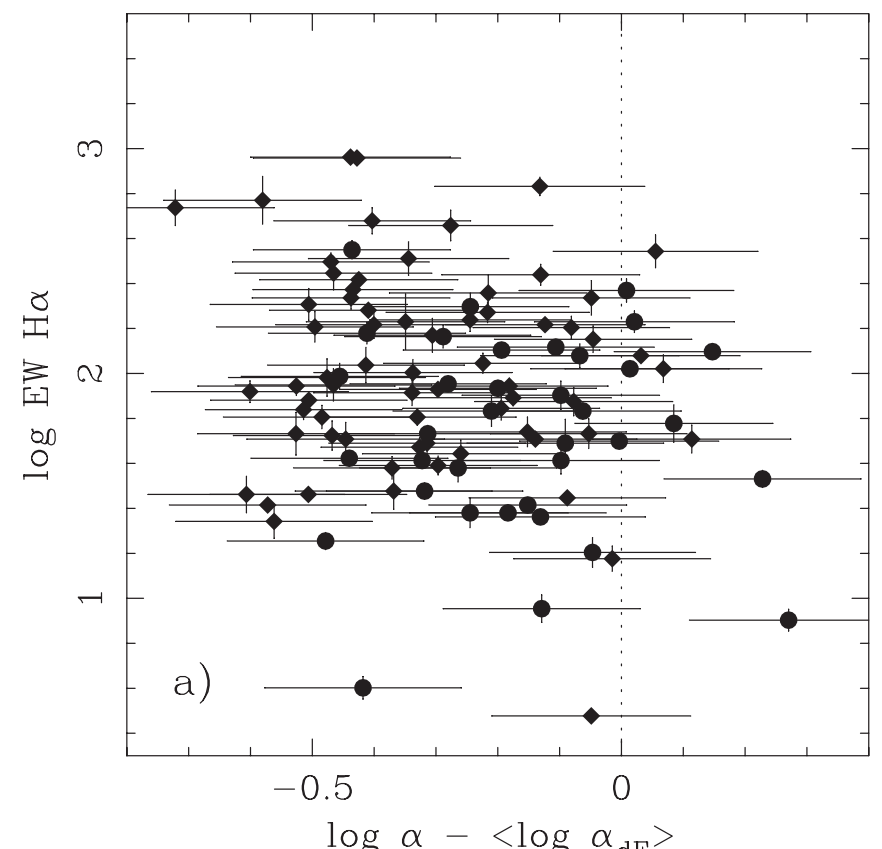

per unit mass of a starburst remains approximately constant for the first few $10^{7} \mathrm{yr}$ and equal to $\sim 10^{35.5} \mathrm{ergs} \mathrm{s}^{-1} M_{\odot}^{-1}$ for a Salpeter IMF and $M_{\text {low }}=1 M_{\odot}$ and $M_{\text {up }}=100 M_{\odot}$ (Leitherer \& Heckman 1995). Thus, equation (5) can be written as

$$
\begin{aligned}
\log (\alpha) & -\left\langle\log \left(\alpha_{\mathrm{dE}}\right)\right\rangle \\
= & \log \left\{1-\frac{\min \left[1,4.84 \times 10^{8} b^{0.55}\left(M_{*} / M_{\odot}\right)^{-1.15}\right]}{1+\psi_{R_{\mathrm{H}}}}\right\},
\end{aligned}
$$

where the burst strength $b$, is the ratio of the stellar mass of the most recent episode of star formation to the galaxy's total stellar mass. Finally, in order to derive the dependence of the color difference $\left[(B-R)-(B-R)_{\mathrm{LSB}}\right]$ on the burst strength we used the predictions of evolutionary synthesis models (e.g., Bruzual \& Charlot 2003). We adopted a 9 Gyr old USP with $Z_{\odot} / 5$ metallicity and a burst of star formation with different burst strengths in the range $b=10^{-4}-1$. For each value of the burst strength we computed the color difference $[(B-R)-(B-$ $\left.R)_{\mathrm{LSB}}\right]$ averaged over the first $10 \mathrm{Myr}$ of evolution of the burst.

Figure $6 b$ shows that in order for the BCD galaxies to show the large degree of expansion measured, their stellar mass should be smaller than $10^{7} M_{\odot}$ and they should have small dark-to-visible mass ratios. Even if the color of the USP were to be much bluer than that of a $9 \mathrm{Gyr}$ old stellar population the vast majority of the galaxies in our sample would be less massive than $10^{8} M_{\odot}$ and the dark matter contribution within the Holmberg radius would be negligible. This is required if the large differences in scale length between BCDs and $\mathrm{dE}$ galaxies are to be explained as due exclusively to the expansion of the underlying stellar mass distribution of BCDs. These conditions are certainly not fulfilled by the galaxies in our sample, where the stellar masses can be much larger than this number $\left(M_{K}\right.$ for our sample can be as high as $-21 \mathrm{mag}$; Paper I) and where significant amounts of dark matter are thought to be present (Ferrara \& Tolstoy 2000). This result again argues against the current star formation having a strong impact on the structural properties of the USP of BCDs. However, detailed studies of individual objects are required to confirm this in all cases.

\section{CONCLUSIONS}

1. We have presented the surface brightness profiles in $B, R$, and $\mathrm{H} \alpha$, for a total of 114 galaxies taken from the Palomar/Las Campanas Imaging Atlas of BCD galaxies. A total 104 of the galaxies are classified as BCDs (see Paper I). The profiles in the continuum bands are characterized by the presence of an HSB component on top of the nearly exponential LSB component associated with the galaxy's USP. At large galactocentric radii the color profiles of $70 \%$ of the galaxies flatten. This flattening occurs approximately at the position where the USP starts to dominate the galaxy surface brightness profile.

2. The color of the USP (corrected for line and nebularcontinuum emission) is systematically redder than the observed integrated color. The color of the USP is bluer than the integrated one only in those objects with the highest equivalent widths of $\mathrm{H} \alpha$ [where the line and nebular-continuum emission is significant; i.e., $\mathrm{EW}(\mathrm{H} \alpha)$ larger that a few hundred angstroms].

3. We find that galaxies with relatively red USP $[(B-R) \geq$ $1 \mathrm{mag}$ ] show structural properties compatible with those of dwarf elliptical galaxies. They show smoother (continuum) light distributions, fainter extrapolated central surface brightness, and this expression yields $F_{0}>1$, the value of $F_{0}$ was set to 1 . For the sake of simplicity we assume the visible mass to be dominated by the galaxy's stellar component. The kinetic luminosity 
larger scale lengths than BCD galaxies with blue envelopes. This result indicates that a non-negligible fraction of the BCD galaxies ( $\sim 15 \%$ ) could be dwarf ellipticals that are now experiencing (or have recently experienced) an episode of star formation.

4. We do not find any correlation between the equivalent width of $\mathrm{H} \alpha$ and the degree of expansion of the USP, the latter being defined as the difference in scale length between BCD and $\mathrm{dE}$ galaxies of identical luminosity. The difference measured between the scale length of BCDs and $\mathrm{dE}$ galaxies is much larger than that expected from changes in the gravitational potential due to the collective effect of supernova-driven winds, especially considering the relatively small differences in $(B-R)$ color between the galaxies and their USP. This suggests that the level of recent star formation in BCDs does not have a significant impact on the structural properties of these galaxies. A detailed study of a large number of individual objects is needed to confirm this.
We are grateful to the Palomar and Las Campanas Observatories staff for their support and hospitality, and to the Caltech/ Palomar and OCIW/Las Campanas Time Allocation Committees for the generous allocation of time to this project. A. Gil de Paz acknowledges financial support from the GALEX mission. A. Gil de Paz is also partially supported by the CONACYT (Mexico) grant 36132-E, the Spanish Programa Nacional de Astronomía y Astrofísica under grant AYA2000-1790, and by NASA through grant HST-AR-10321 from STScI. This research has made use of the NASA/IPAC Extragalactic Database (NED), which is operated by the Jet Propulsion Laboratory, California Institute of Technology, under contract with the National Aeronautics and Space Administration. We would like also to thank K. G. Noeske, C. Sánchez Contreras, and S. Boissier for valuable discussions and to P. Papaderos for providing his compilation of structural properties of dwarf galaxies. We are grateful to the anonymous referee for her/his helpful comments and suggestions.
Barazza, F. D., Binggeli, B., \& Jerjen, H. 2003, A\&A, 407, 121

Binggeli, B., \& Cameron, L. M. 1991, A\&A, 252, 27

Bruzual, A. G., \& Charlot, S. 2003, MNRAS, 344, 1000

Cairós, L. M., Caon, N., Papaderos, P., Noeske, K. G., Vílchez, J. M., GarcíaLorenzo, B., \& Muñoz-Tuñon, C. 2003, ApJ, 593, 312

Cairós, L. M., Caon, N., Vílchez, J. M., González-Pérez, \& Muñoz-Tuñon, C. 2001a, ApJS, 136, 393

Cairós, L. M., Vílchez, J. M., González-Pérez, J. N., Iglesias-Páramo, J., \& Caon, N. 2001b, ApJS, 133, 321

Cannon, J. M., Skillman, E. D., Garnett, D. R., \& Dufour, R. J. 2002, ApJ, 565, 931

Conselice, C. J., Gallagher III, J. S., \& Wyse, R. F. G. 2003, AJ, 125, 66

Crone, M. M., Schulte-Ladbeck, R. E., Greggio, L., \& Hopp, U. 2002, ApJ, 567,258

Crone, M. M., Schulte-Ladbeck, R. E., Hopp, U., \& Greggio, L. 2000, ApJ, 545, L31

Doublier, V., Caulet, A., \& Comte, G. 1999, A\&AS, 138, 213

Doublier, V., Comte, G., Petrosian, A., Surace, C., \& Turatto, M. 1997, A\&AS, 124,405

Drozdovsky, I. O., Schulte-Ladbeck, R. E., Hopp, U., Crone, M. M., \& Greggio, L. 2001, ApJ, 551, L135

Fanelli, M., O’Connell, R. W., \& Thuan, T. X. 1988, ApJ, 334, 665

Ferrara, A., \& Tolstoy, E. 2000, MNRAS, 313, 291

Gil de Paz, A., Madore, B. F., \& Pevunova, O. 2003, ApJS, 147, 29 (Paper I)

Gil de Paz, A., Zamorano, J., \& Gallego, J. 2000a, A\&A, 361, 465

Gil de Paz, A., Zamorano, J., Gallego, J., \& Domínguez, F. de B. 2000b, A\&AS, 145,377

Graham, A., \& Guzmán, R. 2003, AJ, 125, 2936

Hunter, D. A., \& Hoffman, L. 1999, AJ, 117, 2789

\section{REFERENCES}

Jedrzejewski, R. I. 1987, MNRAS, 226, 747

Jerjen, H., Binggeli, B., \& Freeman, K. C. 2000, AJ, 119, 593

Legrand, F. 2000, A\&A, 354, 504

Leitherer, C., \& Heckman, T. M. 1995, ApJS, 96, 9

Loose, H.-H., \& Thuan, T. X. 1985, in Star Forming Dwarf Galaxies and Related Objects, ed. D. Kunth, T. X. Thuan, \& J. T. T. Van (Gif-sur-Yvette: Editions Frontiéres), 73

Mac Low, M.-M., \& Ferrara, A. 1999, ApJ, 513, 142

Noeske, K. G., Guseva, N. G., Fricke, K. J., Izotov, Y. I., Papaderos, P., \& Thuan, T. X. 2000, A\&A, 361, 33

Noeske, K. G., Papaderos, P., Cairós, L. M., \& Fricke, K. J. 2003, A\&A, 410, 481

Papaderos, P., Izotov, Y. I., Thuan, T. X., Noeske, K. G., Fricke, K. J., Guseva, N. G., \& Green, R. F. 2002, A\&A, 393, 461

Papaderos, P., Loose, H.-H., Fricke, K. J., \& Thuan, T. X. 1996a, A\&A, 314, 59 Papaderos, P., Loose, H.-H., Thuan, T. X., \& Fricke, K. J. 1996b, A\&AS, 120, 207

Parodi, B. R., Barazza, F. D., \& Binggeli, B. 2002, A\&A, 388, 29

Schulte-Ladbeck, R. E., Hopp, U., Crone, M. M., \& Greggio, L. 1999, ApJ, 525,709

Schulte-Ladbeck, R. E., Hopp, U., Greggio, L., Crone, M. M., \& Drozdovsky, I. O. 2001, AJ, 121, 3007

Searle, L., Sargent, W. L. W., \& Bagnuolo, W. G. 1973, ApJ, 179, 427

Secker, J., Harris, W. E., \& Plummer, J. D. 1997, PASP, 109, 1377

Silk, J., Wyse, R. F. G., \& Shields, G. A. 1987, ApJ, 322, L59

Tajiri, Y. Y., \& Kamaya, H. 2002, A\&A, 389, 367

Vader, J. P., Vigroux, L., Lachieze-Rey, M., \& Souviron, J. 1988, A\&A, 203, 217

van Zee, L., Salzer, J. J., \& Skillman, E. D. 2001, AJ, 122, 121 